\title{
Particle-laden flows forced by the disperse phase: comparison between Lagrangian and Eulerian simulations
}

\author{
Aymeric Viéa,*, Hadi Pouransari ${ }^{\mathrm{a}, \mathrm{b}}$, Rémi Zamansky $^{\mathrm{c}}$, Ali Mani ${ }^{\mathrm{a}, \mathrm{b}}$ \\ ${ }^{a}$ Center for Turbulence Research, Stanford University \\ ${ }^{b}$ Department of Mechanical Engineering, Stanford University \\ ${ }^{c}$ Institut de Mécanique des Fluides de Toulouse, Toulouse
}

\begin{abstract}
The goal of the present work is to assess the ability of Eulerian moment methods to reproduce the physics of two-way coupled particle-laden turbulent flow systems. Previous investigations have been focused on effects such as preferential concentration, and turbulence modulation, but in regimes in which turbulence is sustained by an imposed external forcing. We show that in such regimes, Eulerian methods need resolutions finer than nominal Kolmogorov scale in order to capture statistics of particle segregation, but gas and disperse phase velocity variances can be captured with resolutions comparable to the Kolmogorov length. The work is then extended to address the question whether Eulerian methods are suitable in scenarios in which the continuum field of interest (temperature or momentum) is itself primarily driven by particles. To this end we have extended our analysis to the problem of turbulence driven by heated particles (Zamansky et al. PoF 2014) and have assessed capabilities of Eulerian methods in capturing particle segregation, as well as statistics of the temperature and velocity fields. Separate investigations are developed for cases with and without buoyancy driven turbulence. For each case corresponding Lagrangian calculations are developed and convergence of statistics with respect to the number of particles is established. Then the statistically-converged Lagrangian and Eulerian results are compared. Results show that accurate capture of segregation by the Eulerian methods always requires resolutions much higher than the nominal Kolmogorov scale. In scenarios for which a continuum phase is forced by particles, results from Eulerian methods show some sensitivity of predicted continuum statistics to the mesh resolution. This sensitivity was found to be largest for the case of a temperature field forced by hot particles, but without presence of buoyancy. In this case a Eulerian method with nominal Kolmogorov resolution was found to be insufficient for capture of temperature statistics. When additional coupling between particles and continuum phase is introduced by including the buoyancy effects, this sensitivity is suppressed in the temperature field, but some sensitivity to the Eulerian mesh resolution were detected in the momentum fields.
\end{abstract}

Keywords: Particle-laden flows; Buoyancy; Radiative heating; Lagrangian particle tracking; Eulerian moment method

\footnotetext{
* Corresponding author

Email addresses: aymeric.vie@centralesupelec.fr (Aymeric Vié), hadip@stanford.edu (Hadi Pouransari), remi.zamansky@imft.fr (Rémi Zamansky), alimani@stanford.edu (Ali Mani)
} 


\section{Contents}

1 Introduction $\quad 3$

2 Simulation methods $\quad 6$

2.1 Particulate phase equations . . . . . . . . . . . . . . . . . . . . 7

2.2 Gas phase equations . . . . . . . . . . . . . . . . . . . . . . 7

2.3 Numerical methods . . . . . . . . . . . . . . . . . . . . . . . . . . 7

2.4 Initial conditions and controlling parameters . . . . . . . . . . . . . . . . . . 8

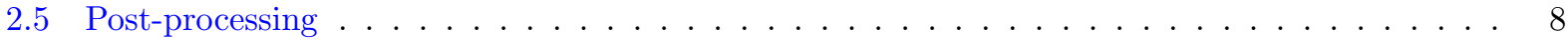

3 Results $\quad 9$

3.1 Decaying turbulence without radiation . . . . . . . . . . . . . . . . . 10

3.1.1 Statistical convergence . . . . . . . . . . . . . . . . . . . . 10

3.1.2 Comparison between Lagrangian and Eulerian results . . . . . . . . . . . . . . . . . 10

3.2 Decaying turbulence with radiation and no gravity . . . . . . . . . . . . . . . . . . 13

3.2.1 Statistical convergence . . . . . . . . . . . . . . . . . . . . . 13

3.2.2 Comparison between Lagrangian and Eulerian results . . . . . . . . . . . . . . . . . 13

3.3 Turbulence with radiation and gravity . . . . . . . . . . . . . . . . . . 15

3.3.1 Statistical convergence . . . . . . . . . . . . . . . . . . 15

3.3.2 Comparison between Lagrangian and Eulerian results . . . . . . . . . . . . . . . . . 18

4 Summary 20

Appendix A Appendix: time signals for case with radiation and gravity 22 


\section{Introduction}

Turbulent particle- or droplet-laden flows play a key role in numerous applications, including natural processes such as droplet clouds, dust storms, and protoplanetary disks, as well as industrial applications such as fuel sprays in internal combustion engines, fluidized beds, particle-based solar receivers, and pharmaceutical sprays. Understanding the key processes underlying the coupled dynamics of particles and fluids in such systems requires development of models capable of reproducing their physics. In most of these systems the particle-laden mixture is under turbulent conditions and can induce preferential concentration in the particle field $[44,12,11,16]$ : inertial particles are ejected from vortex cores and tend to accumulate in low vorticity zones. This phenomenon is characterized by the particle Stokes number, which is the ratio between the particle inertial relaxation time to the Kolmogorov time scale of the turbulence [11].

Previous investigations indicate that preferential concentration is strongest for systems with Stokes number of order unity [11]. Very small particles with small Stokes number essentially follow the flow streamlines, and cannot be effectively centrifuged outside of vortex zones; in the limit of very large Stokes number, the particle phase is hardly influenced by the flow field and thus effects of preferential concentration is suppressed. Preferential concentration plays a key role in various processes including enhancement of particle-particle collision (e.g. leading to faster particle agglomeration or drop coalescence [46, 48]) and turbulence modulation $[19,13,16,4,36]$. In some scenarios preferential concentration plays a primary role even in generation and sustaining turbulence $[52,32]$. Therefore, when it comes to modeling of particle-laden flow phenomena, one key concern is the capability of the model to capture preferential concentration.

Early numerical models attempted to couple Lagrangian particle methods with traditional Eulerian fluid turbulence simulations [37, 12, 43, 44]. In the most simple limit, trajectory of the particles can be determined by use of the Stokes drag formula [45] given the following assumptions:

- The particles are smaller than the Kolmogorov length scale $(d<\eta)$ : particle-resolved DNS is not necessary, and a Point-Particle approximation can be adopted [29].

- The density ratio between the particles and the gas phase is large $\left(\rho_{p}>>\rho_{g}\right)$ : the drag force is dominant over all other forces (added-mass, history, lift).

- the Reynolds number of the particles is smaller than one $\left(\operatorname{Re}_{p}<1\right)$ : the Stokes drag formulation can be used, i.e the momentum relaxation time of the particle is $\tau_{p}=\rho_{p} d^{2} / 18 \mu_{g}$, where $\rho, \mu$, and $d$ denote density, viscosity, and particle diameter respectively, and subscripts "p" and "g" represent particle, and fluid (gas) respectively.

- Dilute regime: the volume fraction is small $\left(\alpha_{p}<10^{-3}\right)$ and thus particle-particle collisions would have negligible impact on primary dynamics.

- Small mass loading $\left(\alpha_{p} \rho_{p} / \rho_{g}<10^{-2}\right)$ : the mass ratio is small enough to avoid momentum two-way coupling between the two phases.

- Monodisperse solid spherical particles: all particles have the same size that does not change with time.

We add to this list another assumption relevant to a subclass of cases with heat transfer:

- Negligible heat capacity for the particle $\left(c_{p, p a r t i c l e}<<c_{p}\right)$ : the heat absorbed by the particles is immediately transferred to the gas phase: there is no need to solve the temperature equation of the particles.

Under such conditions Lagrangian point particle methods have been tested against experiments and were shown to be able to capture the preferential concentration phenomena fairly accurately $[43,12]$. In a typical simulation, the number of numerical particles would be equal to the number of physical particles. Following the aforementioned simplifications, the equations for the Lagrangian particles are limited to their position 
$\mathbf{X}_{p}$ and velocity $\mathbf{V}_{p}$ :

$$
\begin{aligned}
\frac{\mathrm{d} \mathbf{X}_{\mathbf{p}}}{\mathrm{d} t} & =\mathbf{V}_{\mathbf{p}}, \\
\frac{\mathrm{d} \mathbf{V}_{\mathbf{p}}}{\mathrm{d} t} & =\frac{\mathbf{u}_{\mathbf{g}}\left(t, \mathbf{X}_{\mathbf{p}}\right)-\mathbf{V}_{\mathbf{p}}}{\tau_{p}},
\end{aligned}
$$

where $\tau_{p}=\frac{\rho_{p} d^{2}}{18 \mu_{g}}$ is the relaxation time of the particles and $\mathbf{u}_{g}$ is the gas phase velocity.

In the context of mesoscopic DNS simulations, the Lagrangian particle tracking is the reference. However, it still has some limitations. First, if one is aiming to capture the statistics of the disperse, e.g. the local Number Density Function, NDF (number of particles per unit volume), many realizations are needed to develop converged statistics. Additionally, in the limit that the average number of particles per CFD cell is large, Lagrangian methods can become very expensive due to extreme computing clock time needed to track all particles as well as complexities associated with the computational load balancing on parallel machines [18].

Eulerian particle methods have been explored as an alternative to the Lagrangian particle tracking $\operatorname{method}[9,14,15,22,27,28,8,24,47]$. The goal of such methods is to solve the statistics of the disperse phase, i.e. the NDF. Inspired by approaches in kinetic theory of gases [6], the NDF $f\left(t, x, v_{p}\right)$ is defined as the number of particles per unit volume, with certain velocity, $v_{p}$, averaged over many realizations. This NDF satisfies a Population Balance Equation (PBE) (referred to as the Williams-Boltzmann equation in the context of sprays [50]):

$$
\frac{\partial f}{\partial t}+v_{p, i} \frac{\partial f}{\partial x_{i}}+\frac{\partial}{\partial v_{p, i}}\left(\frac{u_{g, i}-v_{p, i}}{\tau_{p}} f\right)=0 .
$$

For an sufficiently large number of realisations of Eq. 2, Eq. 3 is the equivalent of Eq. 2, but written in Eulerian framework. However, to avoid to solve the NDF in the full phase space, moment methods are develop (see for instance [42]), which aim to integrate the PBE over the velocity space to obtain equation on moments, i.e. integrals over the velocity space. The resulting moment equations are [42]):

$$
\begin{gathered}
\frac{\partial C}{\partial t}+\frac{\partial C u_{p, j}}{\partial x_{j}}=0 \\
\frac{\partial C u_{p, i}}{\partial t}+\frac{\partial C\left(u_{p, i} u_{p, j}+\sigma_{i j}\right)}{\partial x_{j}}=C \frac{u_{i}-u_{p, i}}{\tau_{p}}
\end{gathered}
$$

where $C(t, \mathbf{x})$ is the local number density of the particles, $u_{p, i}(t, \mathbf{x})$ is the mean velocity of the particles at the position $\mathbf{x}$, and $\sigma_{i j}(t, \mathbf{x})$ is the covariance matrix of the velocity distribution:

$$
\begin{aligned}
C & =\int f\left(t, \mathbf{x}, \mathbf{v}_{\mathbf{p}}\right) \mathrm{d} \mathbf{v}_{\mathbf{p}}, \\
u_{p, i} & =\frac{1}{C} \int v_{p, i} f\left(t, \mathbf{x}, \mathbf{v}_{\mathbf{p}}\right) \mathrm{d} \mathbf{v}_{\mathbf{p}}, \\
\sigma_{i j} & =\frac{1}{C} \int\left(v_{p, i}-u_{p, i}\right)\left(v_{p, j}-u_{p, j}\right) f\left(t, \mathbf{x}, \mathbf{v}_{\mathbf{p}}\right) \mathrm{d} \mathbf{v}_{\mathbf{p}} .
\end{aligned}
$$

The system of Eq. 4 needs a closure for the covariance matrix of the NDF, based on the underlying physics. A schematic of the possible closure assumptions is depicted in Fig. 1. The two quantities that drive the choice of the model are the particle Stokes number $\mathrm{St}_{k}$ based on the Kolmogorov time scale and the volume fraction [24]. These two quantities control the broadness and shape of the NDF in the velocity space. The Stokes number indicates the occurrence of particle trajectory crossings, i.e. the possibility of multivalued particle velocity at a given space-time instant. For small Stokes number, the particles dominantly follow the fluid and deviation of their velocity from fluid velocity is perturbative (but could be sufficient to induce considerable preferential concentration). It has been shown that for $\mathrm{St}_{k}<1$ a monokinetic assumption, that 


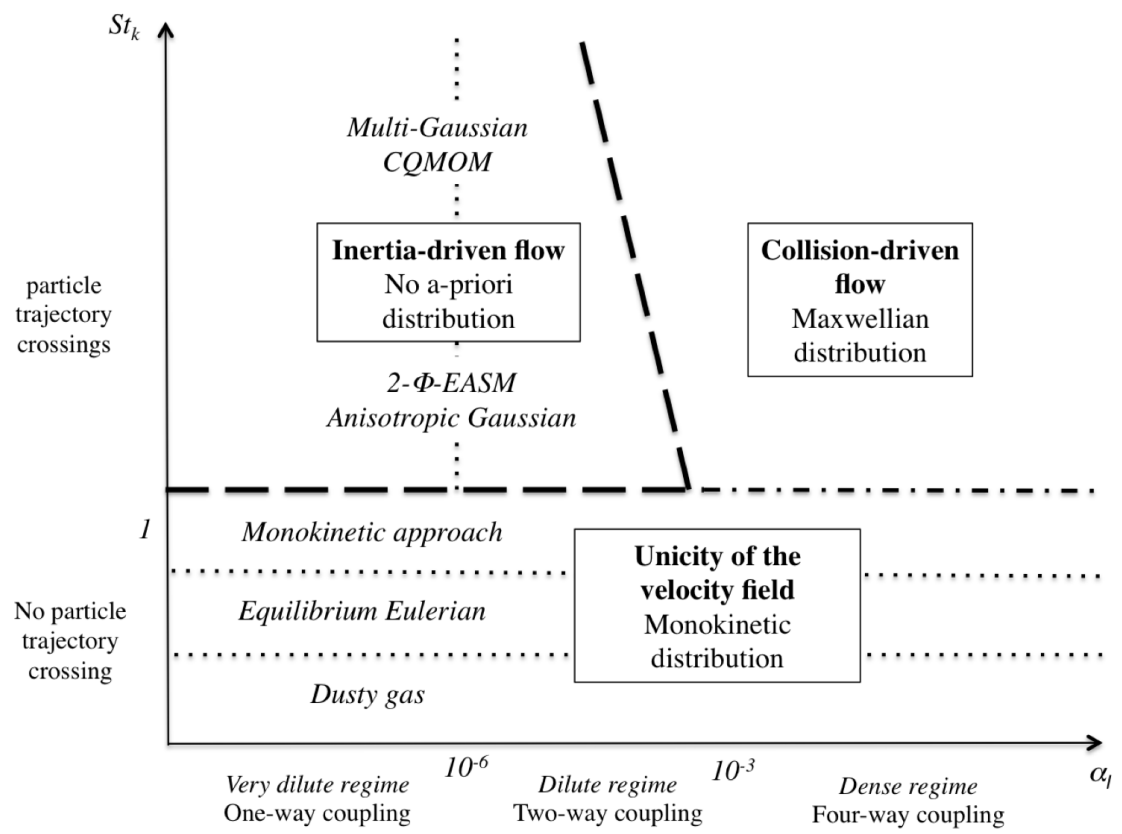

Figure 1: Existence of an a-priori distribution for particle velocity in turbulent flows: for Stokes number below one the particle velocity distribution is Monokinetic. For Stokes number over one and large volume fraction, the velocity distribution is a Gaussian distribution, following the kinetic theory. For Stokes number over one and very dilute to dilute regime, the velocity distribution cannot be anticipated.

is all particles at the same location have the same velocity [23], is indeed valid [3], i.e. only one velocity can describe the particle field per location in physical space. In this range, the covariance matrix can be neglected, and Eq. 4 is closed without any modeling requirement. As classified by Balachandar [2], three approaches of increasing complexity exist in this range of Stokes number:

- Dusty gases $[40,26]$ : the disperse phase velocity is equal to the gas phase velocity. Solely the disperse phase total number density is solved for.

- Equilibrium Eulerian [14, 15]: the disperse phase is evaluated as an expansion around the gas phase velocity. Again, only an equation on the disperse phase total number density is needed.

- Monokinetic approach [9, 23]: the disperse phase velocity is solved through an additional momentum equation, as in Eq. 4.

When $\mathrm{St}_{k}>1$, particles have sufficient inertia to leave the low vorticity regions, and particle trajectory crossings occur [49, 8, 21], which is also referred to as the Random Uncorrelated Motion [17, 21]. In this case when volume fraction of particles is sufficiently large to allow for many collisions, the velocity distribution relaxes towards the Maxwellian distribution, following the kinetic theory [6]. For low volume fraction range assumptions have to be made on the NDF itself (Kinetic-Based Moment Methods, [47, 24]) or on the moment system directly (Algebraic-Closure-Based Moment Methods, [22, 27, 28]).

In the present contribution, we use the Monokinetic Eulerian approach. This way, we will be limited only to the condition of $\mathrm{St}_{k}<1$, which is still relevant to a broad range of applications. We then focus on addressing two sources of error of this approach under such conditions:

- The statistical convergence: the Eulerian approach solves the infinite-realization limit of the Lagrangian particle system, i.e. the Eulerian simulation is the sum of all possible Lagrangian realizations of the disperse phase. In this context we first ask the question: how many particles are needed to allow for a Lagrangian disperse phase to be accurately represented by a Eulerian model? Secondly, 
in a two-way coupled context, each Lagrangian realization could affect the carrier phase differently, possibly leading to a different evolution for the gas phase statistics. However the Eulerian model can capture only the mean effect of many Lagrangian ensembles on the gas phase. Consequently we ask the question: is the gas phase of the Eulerian simulation equivalent to the one of an individual particle realization of the reference Lagrangian simulation?

- The numerical resolution: actually, if statistical convergence and small Stokes number conditions are fulfilled, the Monokinetic Eulerian Method and the Lagrangian approach are equivalent and will eventually lead to the same results. However, solving the Eulerian equations numerically will introduced a numerical bias, that will eventually disappear for an infinite mesh resolution in the case of consistant and stable numerics. Consequently, one of the main issues of the Eulerian approach is the design of numerics, as the disperse phase can exhibit large gradients and vacuum zones. Realizable capturing of these extreme conditions inevitably requires numerical methods with inherent dissipation. In this context we ask the question: how fine should be the Eulerian grid to allow for accurate representation of particle and flow field statistics?

In this paper we have investigated these questions in particle-laden fluid mixtures in a series of regimes with increasing complexity in terms of coupling between the two phases. We first consider the case of decaying homogeneous isotropic turbulence, which has been studied extensively in the literature. Here we investigate it because it isolates the effect of the gas phase on the disperse phase through drag force, without retro-coupling of the disperse phase to the gas phase. It will help us to first highlight the level of comparison between Lagrangian and Eulerian methods in an intrinsically one-way coupled system. By establishing a rough criterion for the resolution of Eulerian methods that would capture converged statistics of particles, we set the stage for testing of more complex systems. We will consider next the case of a decaying turbulence subject to radiative heating. In this second test case, the particles receive radiation, and consequently heat the gas phase. However, as no gravity force is taken into account, no buoyancy effect is generated, and the turbulence is still decaying, and is weakly modified by dilatation effects. The goal of this test case is to assess the consequences of the accuracy of the prediction of the particle segregation on the temperature variance. Lastly, we will present results for the case of radiatively heated particle laden flow subject to gravity and buoyancy effects. In this case, heating of the fluid by the particles can generate and sustain turbulence [52]. In other words, the particle field, and specifically particle segregation, is the main driver of turbulence in the long run. We will present an analysis on whether the error in the particle segregation due to underresolved Eulerian method would have consequences in the prediction of the turbulence itself. The paper is then concluded by presenting a summary of our general recommendations for simulation of particle-laden flows.

\section{Simulation methods}

Before describing the results of Eulerian and Lagrangian methods, we briefly explain the computational methods and aspects of the model problem used in this study. We have considered a triply periodic box of homogeneous turbulence laden with particles with zero mean flow. Three separate investigations are carried out to study the regimes of 1) decaying turbulence one-way coupled with particles, 2) radiatively heated particles in decaying turbulence without buoyancy, and 3) buoyancy driven turbulence via radiatively heated particles in a particle-fluid mixture. Below we describe the governing equations considered, the employed numerical methods, initial conditions, and the post-processing methodology. 


\subsection{Particulate phase equations}

Considering the Monokinetic assumption, the Eulerian Moment method equation for the dimensionless concentration $C$, and velocity $u_{p, i}$ are [23]:

$$
\begin{aligned}
\frac{\partial C}{\partial t}+\frac{\partial C u_{p, j}}{\partial x_{j}} & =0 \\
\frac{\partial C u_{p, i}}{\partial t}+\frac{\partial C u_{p, i} u_{p, j}}{\partial x_{j}} & =C \frac{u_{i}-u_{p, i}}{\tau_{p}} .
\end{aligned}
$$

\subsection{Gas phase equations}

The equations for dimensionless gas density $\rho$, velocity $u_{i}$ and temperature $T$ are:

$$
\begin{aligned}
\frac{\partial \rho}{\partial t}+\frac{\partial \rho u_{j}}{\partial x_{j}} & =0 \\
\frac{\partial \rho u_{i}}{\partial t}+\frac{\partial \rho u_{i} u_{j}}{\partial x_{j}} & =-\frac{\partial P}{\partial x_{i}}+\frac{1}{\operatorname{Re}} \frac{\partial}{\partial x_{j}}\left(\frac{\partial u_{i}}{\partial x_{j}}+\frac{\partial u_{j}}{\partial x_{i}}-\frac{2}{3} \delta_{i j} \frac{\partial u_{k}}{\partial x_{k}}\right)+(\rho-1) \frac{\delta_{i 3}}{\mathrm{Fr}^{2}}, \\
\frac{1}{\gamma} \frac{\partial \rho T}{\partial t}+\frac{\partial \rho T u_{j}}{\partial x_{j}} & =\frac{1}{\operatorname{RePr}} \frac{\partial}{\partial x_{i}}\left(\frac{\partial T}{\partial x_{i}}\right)+\alpha(C-1),
\end{aligned}
$$

where $1 / \operatorname{Re}$ is the dimensionless viscosity, $\operatorname{Pr}$ is the Prandtl number, $\gamma$ is the ratio of specific heats, and Fr is the Froude number. The radiative heat flux parameter $\alpha$ represents the dimensionless radiative heat received per particle in an optically thin mixture in which particles are the main absorbers of radiation. Density and temperature are linked through the dimensionless equation of state $\rho T=1$. The term involving $-1 / \mathrm{Fr}^{2}$ in the momentum equation represents the hydrostatic pressure gradient. Here we have included this term explicitly, to allow for use of periodic assumption in the remaining portion of the pressure field. The term $-\alpha$ in the energy equation stands for a background heat loss to the environment in our model problem to avoid global energy accumulation and allow for temporally stationary regimes with well-defined statistics. Zamansky et al. [52] rigorously arrived at a similar formulation for the energy equation by deriving an equation for temperature fluctuations about a slowly growing mean in the framework of the Boussinesq approximation. Looking at the system of equations, it is clear that under the set of assumptions we are considering, the particulate phase is only affecting the gas phase through its concentration. Its mass loading has no impact because it is considered to be small enough to neglect its effect. However, it has to be kept in mind that studying the effect of this parameter would be of great importance for two-way coupled systems outside of the limits we investigate here. As a consequence, for the Lagrangian particle tracking, the concentration field must be computed to close Eqs. 12.

\subsection{Numerical methods}

The gas phase equations are solved using the Low-Mach number approximation [7, 20]. Spatial derivatives are evaluated through second order central differences. The variable coefficient Poisson equation for aerodynamic pressure is solved using FFT (Fast Fourier Transform) in an iterative procedure to account for variation in density and dilatation effects. For the time integration of the Eulerian moment methods, a second order scheme is used $[5,8]$. The integration of the source terms is performed using a second order Strang Splitting. The limiter used for slope evaluation is a double minmod [38, 39], to handle the large gradients in the particle concentration and velocity fields.

Concerning the Lagrangian tracking, a fourth order Runge-Kutta method is used to solve Eq. 2. Lastly, we use a second order linear interpolation scheme for gas phase-disperse phase exchanges [1]. More complex and more accurate strategies exist in the literature and would impact the statistics of the disperse phase, see $[30,25]$ for example. However we have chosen this method because it is widely used in the literature (see for instance $[35,41]$ ) because of its simplicity and the fact that it is straightforward to implement in unstructured code [31]. This choice would impact the results on disperse phase statistics, but it will be seen that for comparison with Eulerian solutions, the level of bias introduced in the Lagrangian results will not modify the conclusions. 


\subsection{Initial conditions and controlling parameters}

In all of the presented test cases, the ratio of the specific heats, $\gamma$, is chosen to be equal to 1.4, and the Prandtl number, $\mathrm{Pr}$, is set to be equal to 1.0. Other parameters that we chose in order to initiate our test cases are:

- the turbulent Reynolds number $\mathrm{Re}_{t}$ is set by the initial condition of the turbulence. Here we use a Passot-Pouquet spectrum [34] with a Reynolds number based on the Taylor microscale $\operatorname{Re}_{\lambda}=27$ with a total kinetic energy equal to 3 and a dimensionless viscosity $1 / \operatorname{Re}=1.4610^{-3}$. As no artificial forcing is considered, the turbulent energy will decay if no physical forcing is involved.

- the radiative heat flux parameter $\alpha$ represents the radiative heat absorption per reference particle concentration per reference time. In the chosen dimensionless form, $\alpha$ also represents the ratio of heating time rate to the eddy turnover frequency. Depending on the test case, we chose $\alpha$ to be equal to zero or 0.1 , respectively corresponding to non-heated and mildly heated regimes.

- the relaxation time of the particles $\tau_{p}$, directly impacts the preferential concentration phenomenon, by setting the level of inertial of the particles [11]. $\tau_{p}$ is a key parameter because it will determine whether the adopted Eulerian approach is suitable or not. In the following, we will consider $\tau_{p}=0.01$, $0.025,0.05,0.10,0.20,0.40$ and 0.80 . This dimensionless relaxation time is linked to the classical Stokes number based on Kolmogorov time scale $\mathrm{St}_{k}=\tau_{p} / \tau_{k}$. For the cases with decaying turbulence, the reference dimensionless Kolmogorov time based on initial conditions is measured to be equal to $\tau_{K}=0.1$. For the cases in which particle heating sustains the turbulence, the Kolmogorov time and particle Stokes number are computed as part of the post processing.

- the size of the domain: previous investigations have explored box-size dependence of statistics in particle-laden flows $[52,51]$. In the present study, as our goal is mainly to compare Eulerian and Lagrangian approaches, we will not investigate this issue, and will consider the standard domain size equal to $L_{b o x}=2 \pi$. Combining the Reynolds number and the domain size, the nominal mesh size for resolving the turbulence at initial conditions is $64^{3}$ cells [33].

- initial particle distribution: particles are uniformly distributed at time $t=0$. For the Lagrangian particle tracking, they are randomly drowned over the entire space whereas for the Eulerian simulations, the initial concentration is set to $C(t, \mathbf{x})=1$. In terms of number of particles, as we are interested in the statistical convergence, we investigate cases with average particle number per cell equal to $1,4,16,64$ and 256 , i.e. $0.262 \mathrm{M}, 1 \mathrm{M}, 4.1 \mathrm{M}, 16.7 \mathrm{M}$ and $67 \mathrm{M}$ particles in the whole domain ${ }^{1}$. In all our simulations, each numerical particle corresponds to a physical particle, as we are not using a parcel method like in [10]. We are not adjusting the mass loading when changing the number of particles, as we are considering the very dilute regime in which it has no effect on the system. All simulations start with a particulate phase at rest, i.e. $\mathbf{u}_{p}(t=0, \mathbf{x})=0$ or $\mathbf{V}_{p}(t=0)=0$.

\subsection{Post-processing}

To analyse each test case, we investigate macroscopic quantities obtained by spatial averaging over the domain $\langle\cdot\rangle$. For the gas phase, we focus on the Turbulent Kinetic Energy (TKE) of the gas phase $\left\langle u_{g, i} u_{g, i}\right\rangle$, as a measure of the turbulence level, and the temperature variance $\left\langle T_{g}^{\prime 2}\right\rangle=\left\langle T_{g}^{2}\right\rangle-\left\langle T_{g}\right\rangle^{2}$, as a measure of the effect of segregation on the heat exchange between the two phases.

For the disperse phase, we investigate two statistical quantities: The Turbulent Kinetic Energy of the particle phase (PTKE), as a measure of the effect of the turbulence level on the dynamics of the particles, and the segregation of the particle phase as a measure of preferential concentration effects due to the turbulence.

\footnotetext{
${ }^{1}$ Note that in the temperature equation, the dimensionless particle concentration is used, so that the overall energy input is the same regardless of the number of particles.
} 
The disperse phase quantities are evaluated in a different way for each modeling approach. For the Lagrangian particle tracking method, the PTKE is evaluated as the average over all particles:

$$
\mathrm{PTKE}=\frac{1}{N_{p}} \sum_{k=1}^{N_{p}} \frac{V_{k, i} V_{k, i}}{2}
$$

whereas in the Eulerian method, PTKE is defined as $\left\langle u_{p, i} u_{p, i}\right\rangle$.

Segregation is defined as the normalized mean square concentration. However, given that for the Lagrangian particles, definition of concentration is scale-dependent, a reference projection scale needs to be defined. In [47], the authors used a reference mesh as a projection for mesh for all Lagrangian and Eulerian quantities. Here we adopt the nominal Kolomogorov-resolved mesh for the initial conditions of size $64^{3}$ as the reference mesh for projection of concentration. Consequently, we define a projected particle number density as:

$$
C_{\mathrm{Lag}}(t, \mathbf{x})=\frac{1}{N_{p} \delta_{E}^{3}} \sum_{k=1}^{N_{p}} \int \delta\left(\mathbf{x}-\mathbf{X}_{k}\right) \mathcal{H}\left(\mathbf{x} \mid \delta_{E}\right) \mathrm{d} \mathbf{x},
$$

where $\mathcal{H}$ is the cubic hat function centered at $\mathbf{x}$ and of characteristic width $\delta_{E}$. In our simulations, $\delta_{E}$ is equal to the reference grid spacing of the Lagrangian simulations, i.e., $\delta_{E}=2 \pi / 64$. It follows that the Lagrangian evaluation of the segregation is:

$$
g_{p p}^{\mathrm{Lag}}=\frac{\left\langle C_{\mathrm{Lag}}^{2}\right\rangle}{\left\langle C_{\mathrm{Lag}}\right\rangle^{2}} .
$$

For the Eulerian approach, to be consistent with the Lagrangian method, the concentration field is also projected onto the reference mesh of the Lagrangian simulations and then the segregation is evaluated:

$$
g_{p p}^{\text {Eul }}=\frac{\left\langle C_{\text {proj }}^{2}\right\rangle}{\left\langle C_{\text {proj }}\right\rangle^{2}},
$$

where $C_{\text {proj }}$ is the projected concentration field. We shall note that, even for a statically uniform distribution for the Lagrangian particle, the segregation is not equal to one exactly. Since particles are randomly and independently drawn, the local number of particles follows the binomial distribution. Thus one can show that the segregation for a homogeneous Lagrangian sampling is $g_{p p}=(\lambda+1) / \lambda$ where $\lambda$ is the average number of particles per cell. Consequently the initial segregation of the Lagrangian computations are $g=2,1.25,1.062,1.015$ and 1.003 for 1, 4, 16, 64 and 256 particles per cell. At this point, it is worth noticing the way we evaluate concentration for the post-processing differs from the one we use for the simulation (cubic hat versus linear interpolation). Here we follow the work of Kaufmann et al. [22], in which the authors compared different projection strategies for transforming Lagrangian quantities into Eulerian fields for the sake of comparison (not for the numerical implementation). They finally demonstrates that Gaussian and Cubic hat filters have equivalent accuracy for evaluating such Eulerian field.

\section{Results}

In the following, we present analysis of simulations of particle-laden flow under the aforementioned three regimes. For each case, we first evaluate the statistical convergence level of the Lagrangian computations. In this stage, by systematically increasing the number of Lagrangian particles, we determine the minimum number of particles that would result in statistics independent of particle numbers. In such limit, the present Eulerian moment method would be well defined. Second, by using statistically converged Lagrangian solutions as a reference, the accuracy of the macroscopic Eulerian results and their dependence on resolution is analyzed. Both Lagrangian and Eulerian calculations are performed over a wide range of parameters by varying the particle Stokes number. 


\subsection{Decaying turbulence without radiation}

\subsubsection{Statistical convergence}

The first test case is the decaying turbulence without radiation, for which no forcing is active on turbulence. First, we investigate the statistical convergence of the Lagrangian method. Fig. 2 shows the time evolution of the segregation of the particles, and the PTKE for different particle concentrations and different relaxation times. One can see that the number of particles results is not sufficient to reach a converged statistics in segregation. For these cases, even at the initial condition where the particles are homogeneously distributed, the computed segregation is already at 2, which is an expected result given that there is not enough particle to "feed" each cell, thus leading to "holes" in the concentration field. This is expected as the random uniform distribution will follow a binomial distribution and will have fluctuations that goes at the square root of the number of particles. By increasing the number of particles, a unity initial segregation is recovered, showing the statistical convergence. The predicted PTKE on the other hand, is insensitive to the number of particles in the domain. This is expected since computation of PTKE does not require projection over a subdomain scale. Instead, the kinetic energy of all particles is directly averaged over the entire domain that involves sufficient number of particles for all cases. We should note that in these one-way coupled systems particle-particle interactions, and particle-fluid interactions are absent, and thus each particle represents an independent realization of PTKE.

Fig. 3 more effectively summarizes these results, by plotting the maximum (in time) of both segregation and PTKE as a function of particle relaxation time ${ }^{2}$. These results confirm that statistically converged segregation is approximately achieved if using at least $16 \mathrm{M}$ particles, which is equivalent of 64 particles per CFD cell. It can also be noticed that the statistical bias induced by the finite number of particle on the segregation is constant over all simulations. Consequently, it will have less impact on simulations with high segregation, as the error will become marginal compared to low segregation cases.

\subsubsection{Comparison between Lagrangian and Eulerian results}

Next, we present results from Eulerian simulation of particle-laden flows under the same conditions as in the Lagrangian calculations. Fig. 4 shows segregation and PTKE versus time for different mesh resolutions and in comparison to the corresponding Lagrangian results with $N_{p}=64$ per cell. We note again that the Eulerian segregation results are computed after projection of concentration to the same reference mesh used in the Lagrangian method.

For small relaxation time $\left(\mathrm{St}_{k}<<1\right)$ the preferential concentration is weak and segregation deviates only slightly from unity. However, correct prediction of this small deviation requires refined Eulerian simulations. For moderate Stokes number, preferential concentration is highly active, and segregations deviates significantly from unity. While in relative sense convergence with mesh refinement is similar to that of low Stokes regimes, the absolute errors are considerable, even with the finest Eulerian resolutions. It can also be noticed that the apparent order of convergence is one, where the numerics used are at second order. This is because of the slope limiter that is required to ensure a stable and robust numerical methods, but on the other hand smears out gradients and accumulations. It consequently affects the order of accuracy on the segregation, as the limiter will drive the numerics to first order accuracy in these regions. The PTKE value however, are insensitive to the mesh resolution and Eulerian simulations match with Lagrangian simulations.

These conclusions are confirmed by Fig. 5, where maximum segregation and PTKE are plotted against particle relaxation time. Mesh convergence is shown for relaxation time below 0.1, i.e. Stokes number below one. For larger Stokes number the monokinetic model used here will not be valid and generally overestimate segregation, due to missing the trajectory crossing phenomenon, which is active in the higher Stokes regime. The PTKE results show a small sensitivity to mesh size, but results are still satisfactory for every mesh refinement.

\footnotetext{
${ }^{2}$ For the high Stokes number case, the maximum segregation is achieved for the latest time which is $\mathrm{t}=10$ in our simulation, and is not visible in Fig. 3
} 

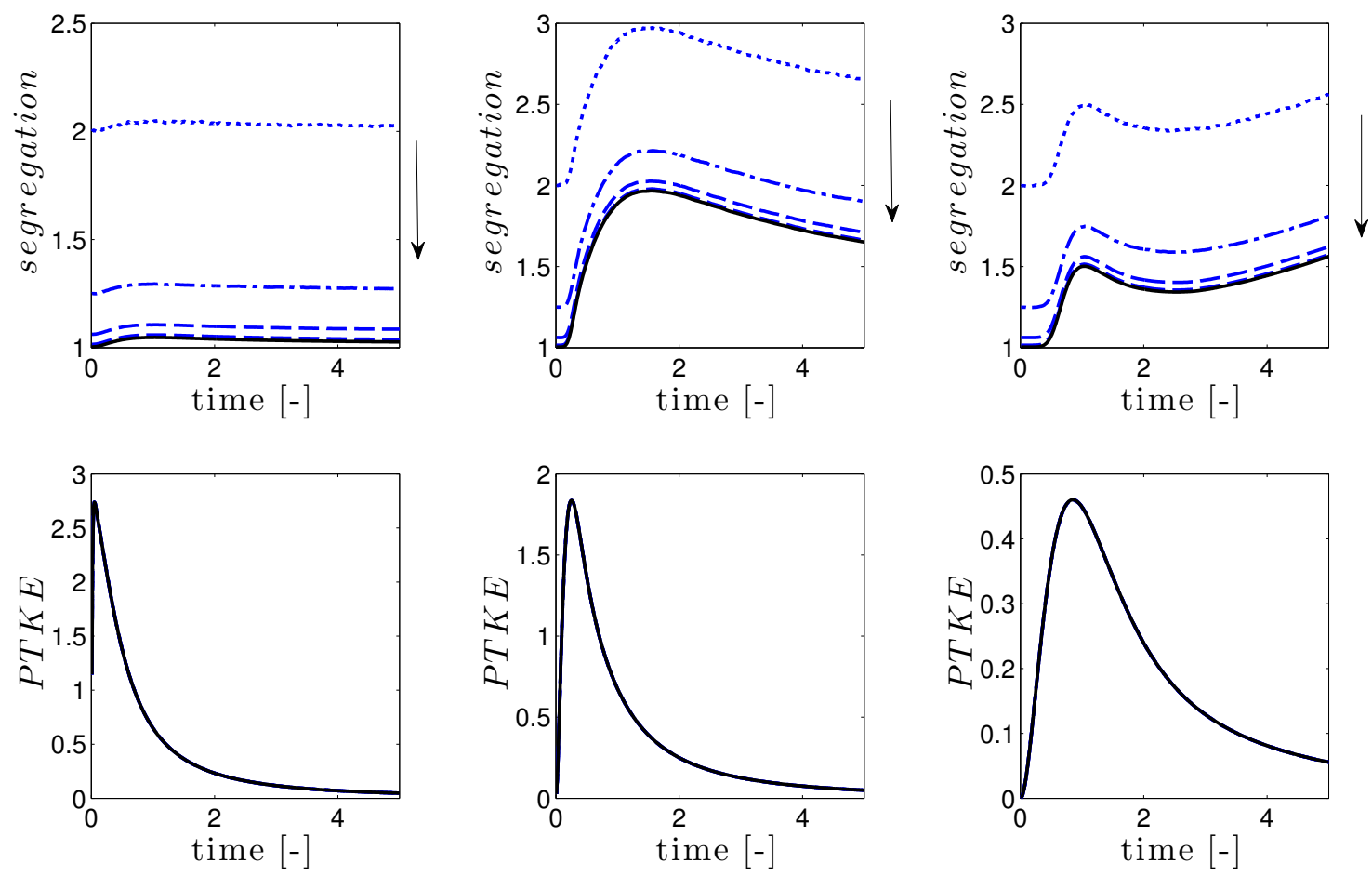

Figure 2: Decaying turbulence without radiation: effect of the number of particles on the statistics of the Lagrangian simulations. Segregation (top) and Particle Total Kinetic Energy (bottom) versus time for $\tau_{p}=0.01,0.1$ and 0.8 . The arrows indicate increasing number of particles by a factor of 4 from 1 particle per cell to 256 particles (black full line) per cell. For the PTKE, all the results are overlapping.
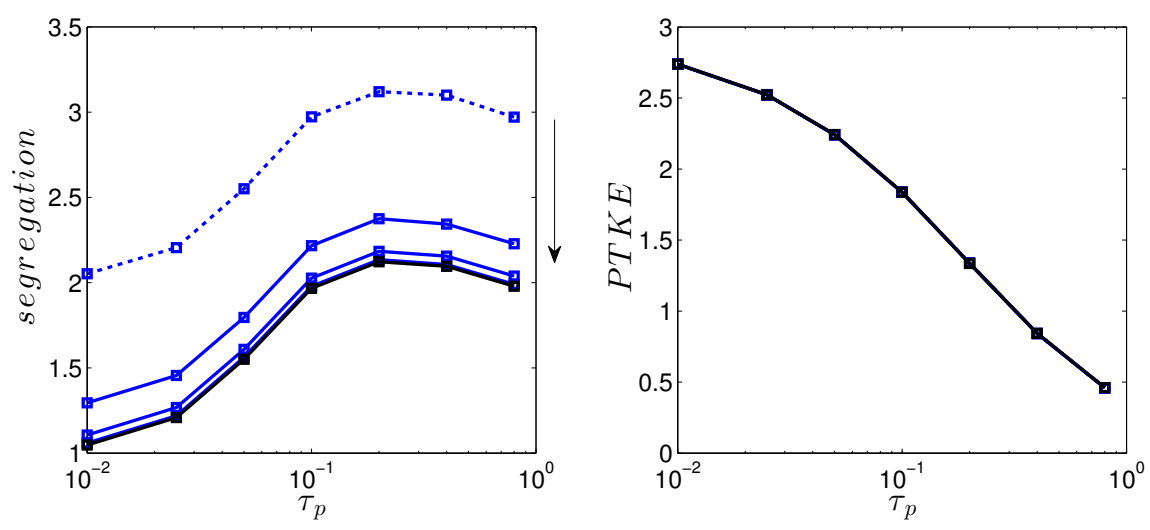

Figure 3: Decaying turbulence without radiation: effect of the number of particles on the statistics of the Lagrangian simulations. Maximum segregation (left) and Particle Total Kinetic Energy (right) versus $\tau_{p}$. The arrow indicates increasing number of particles by a factor of 4 from 1 particle per cell to 256 particles (black full line) per cell. For the PTKE, all the results are overlapping. 

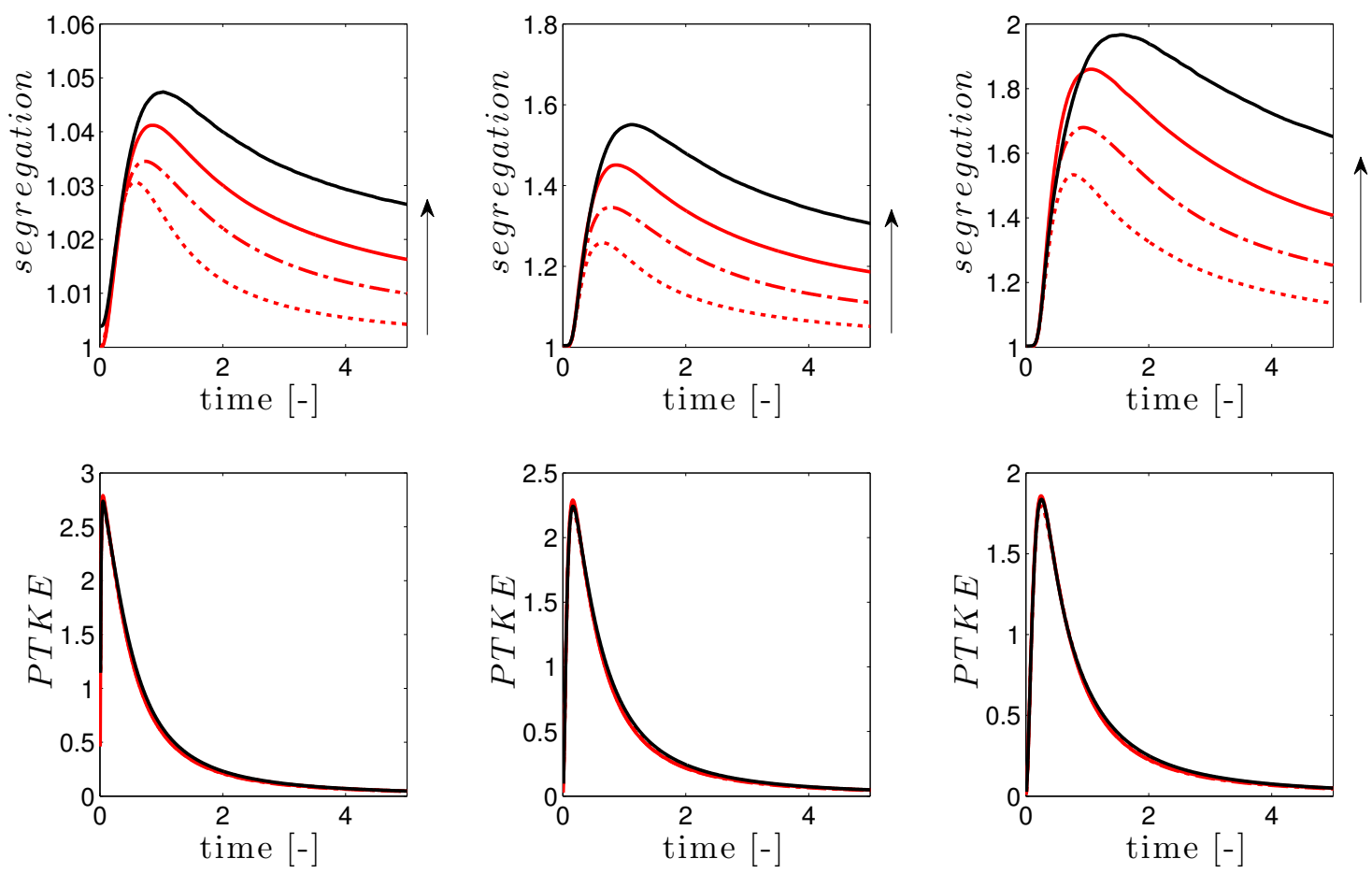

Figure 4: Decaying turbulence without radiation: Lagrangian with 64 particles per cell (black full) versus Eulerian (red) simulations. Segregation (top) and Particle Total Kinetic Energy (bottom) versus time for $\tau_{p}=0.01,0.05$ and 0.1 . The arrows indicate increasing mesh size from $64^{3}$ to $256^{3}$.
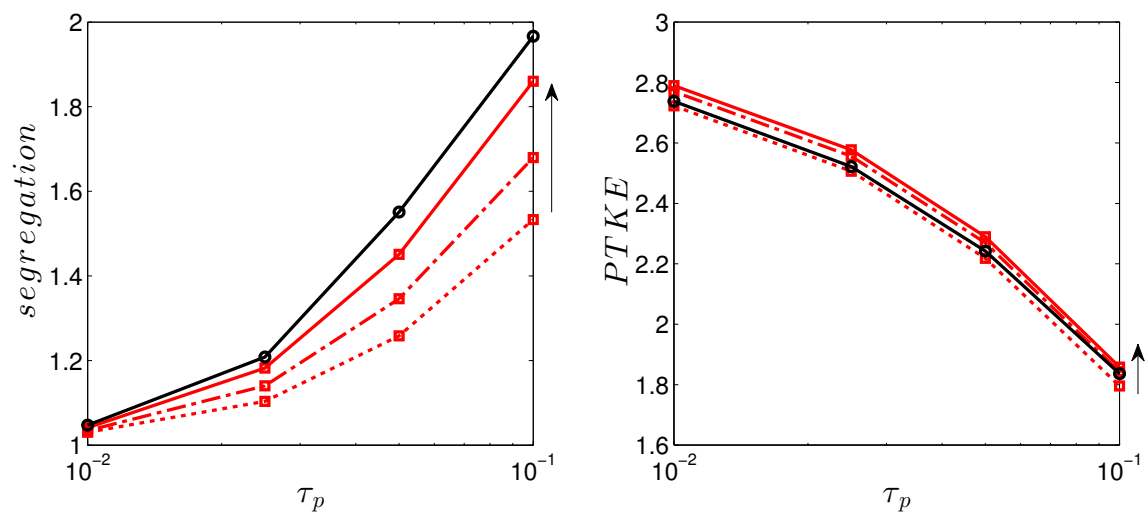

Figure 5: Decaying turbulence without radiation: Lagrangian with 64 particles per cell (black full) versus Eulerian (red) simulations. Maximum segregation (left) and Particle Total Kinetic Energy (right) versus $\tau_{p}$. The arrows indicate increasing mesh size from $64^{3}$ to $256^{3}$. 


\subsection{Decaying turbulence with radiation and no gravity}

\subsubsection{Statistical convergence}

As we turn on the radiative heating of the particles, they will transfer energy to the gas phase, and hence they generate temperature inhomogeneities. Therefore, this case presents the first example for investigation of the temperature statistics and examining whether misprediction of segregation can lead to misprediction in heat transfer.

When buoyancy is absent (i.e. no gravity), the heat transfer primarily contributes to dilatation modes of fluid motion and not much of vorticity production, as the density and pressure gradients are almost aligned. The TKE decay remains similar to the regime without radiation and insensitive to the number of particles, therefore we avoid presenting TKE, and PTKE plots for this case.

In Fig. 6 segregation and temperature variance are shown as a function of time. Plots are repeated with increasing the number of particles (while decreasing $\alpha$ proportionally to maintain the same heating levels), and for different particle relaxation times. The statistical convergence of the preferential concentration follows the same trend as without radiation.

A comparison between the case without radiation and the one with radiation indicates that segregation has been suppressed as a result of radiation. This is expected, because heating, which is stronger in regions with higher particle concentration, acts to expand the gas locally. Subsequently, the particle field is expanded because particles follow the gas, and the imposed divergence lowers the particle concentration in these regions.

Given the direct connection between heat transfer and particle concentration, one expects that the convergence of temperature statistics is tied to that of concentration. However, our results, presented in Fig. 6, show only limited sensitivity of temperature variance to the number of particles per cell, with sensitivity becoming weaker at higher Stokes numbers. While various complex physical and numerical effects can be contributing to these trends, we briefly note two effects.

The first effect is associated with the fact that temperature is driven by integral of heat source in time and not heat source itself. At higher Stokes numbers motion of fluid is less correlated with that of particles, and thus a fluid element is likely to sample concentration fields from a neighborhood with its temperature representing a locally averaged concentration effect. This Stokes-dependent smearing can explain lower levels of variability in RMS of temperature compared to that in concentration itself. The second effect is due to the fact that heat transfer has an inherent physical diffusion, which also acts to smear out thermal fields. When the particle-particle distance becomes smaller than this smearing length (which can be larger than mesh size), because of the preferential concentration [53], the temperature field recognizes the particles field as a continuum and establishes convergence in thermal statistics.

Fig. 7 presents a summary of the results in Fig. 6 by showing the maximum (in time) segregation and temperature variance as a function of particle relaxation time. It turns out that for particle relaxation times above 0.04 (corresponding to Stokes number of 0.4) the computed temperature variance is barely sensitive to the number of particles, as long as the average particle per cell is larger than one.

\subsubsection{Comparison between Lagrangian and Eulerian results}

We here present the first analysis that assesses the resolution requirements of a Eulerian particle method for capturing scalar fields such as temperature. Again, we avoid presenting statistics of PTKE, since these are well converged due to the reasons described in section 3.1.

In Fig. 8, the segregation and temperature variance are compared between Lagrangian and Eulerian simulations for different particle relaxation times. Given the limitation of the monokinetic Eulerian method to Stokes below unity, we still performed Eulerian calculations only up to $\tau_{p}=0.1$. Like the case without radiation, the segregation is underestimated, even for resolutions four times the single-phase DNS (i.e. $256^{3}$ mesh), but the statistics consistently converge to that of Lagrangian calculation with mesh refinement. The figure also shows the direct consequence of segregation mismatch on the predicted Eulerian temperature variance.

It turns out that temperature statistics are significantly more sensitive to mesh resolution in the Eulerian method, than to number of particles in the Lagrangian calculations. This observation suggests that the 

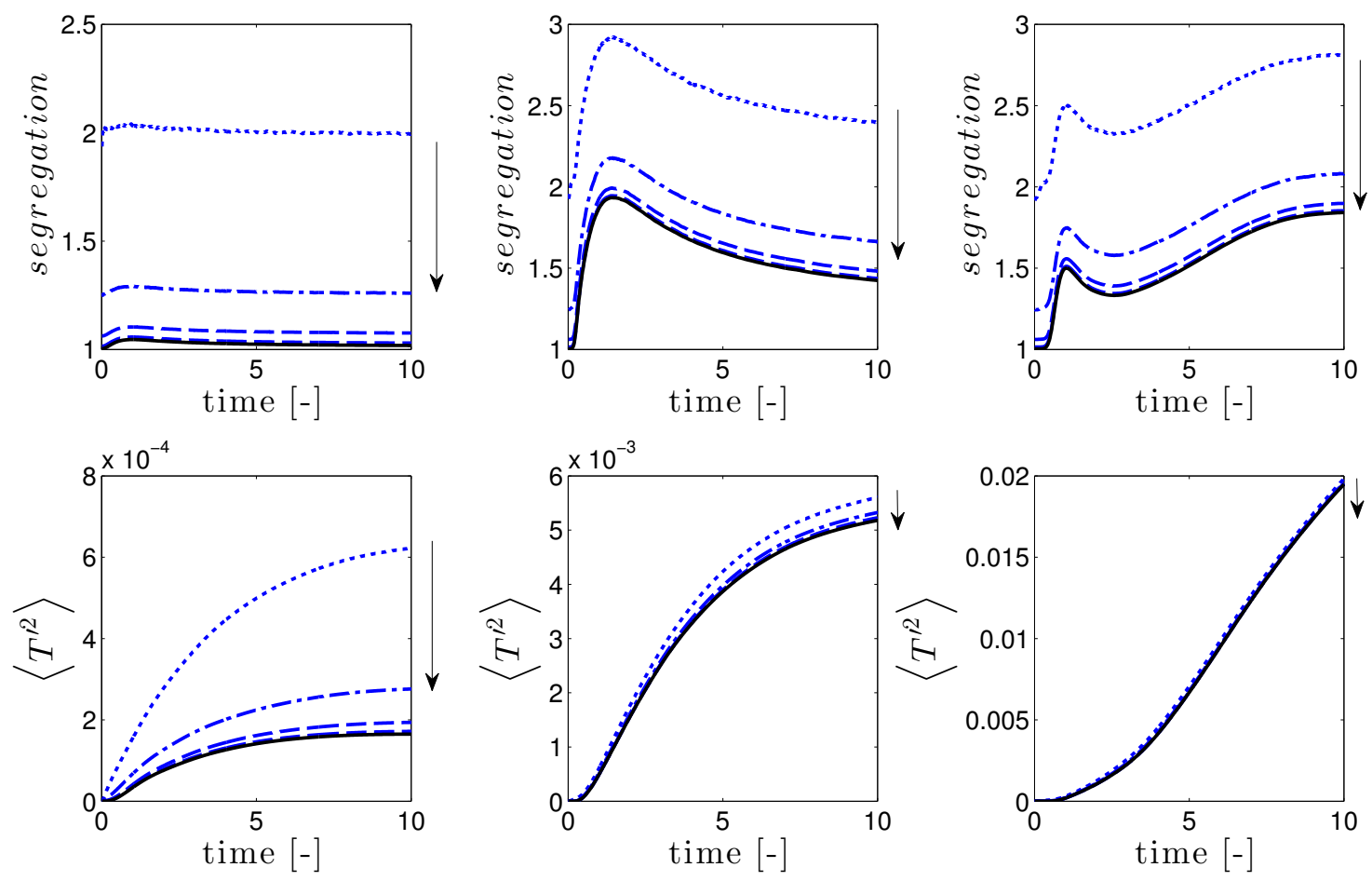

Figure 6: Decaying turbulence with radiation and no gravity: effect of the number of particles on the statistics of the Lagrangian simulations. Segregation (top) and Temperature variance (bottom) versus time for $\tau_{p}=0.01,0.1$ and 0.8 . The arrows indicate increasing number of particles by a factor of 4 from 1 particle per cell to 256 particles (black full line) per cell.
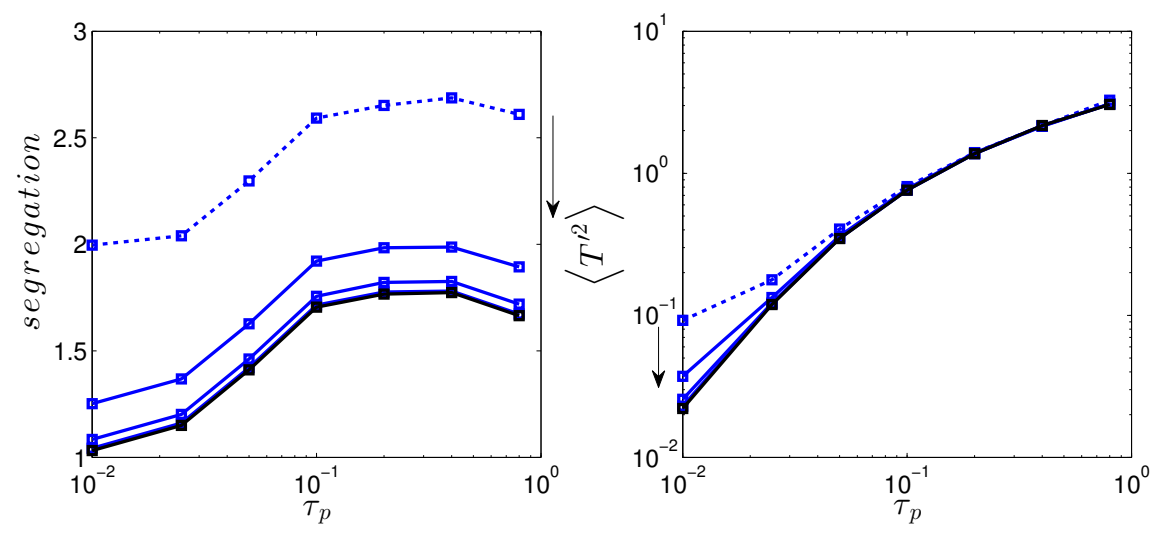

Figure 7: Decaying turbulence with radiation and no gravity: effect of the number of particles on the statistics of the Lagrangian simulations. Maximum segregation (left) and Temperature variance (right) versus $\tau_{p}$. The arrows indicate increasing number of particles by a factor of 4 from 1 particle per cell to 256 particles (black full line) per cell. 

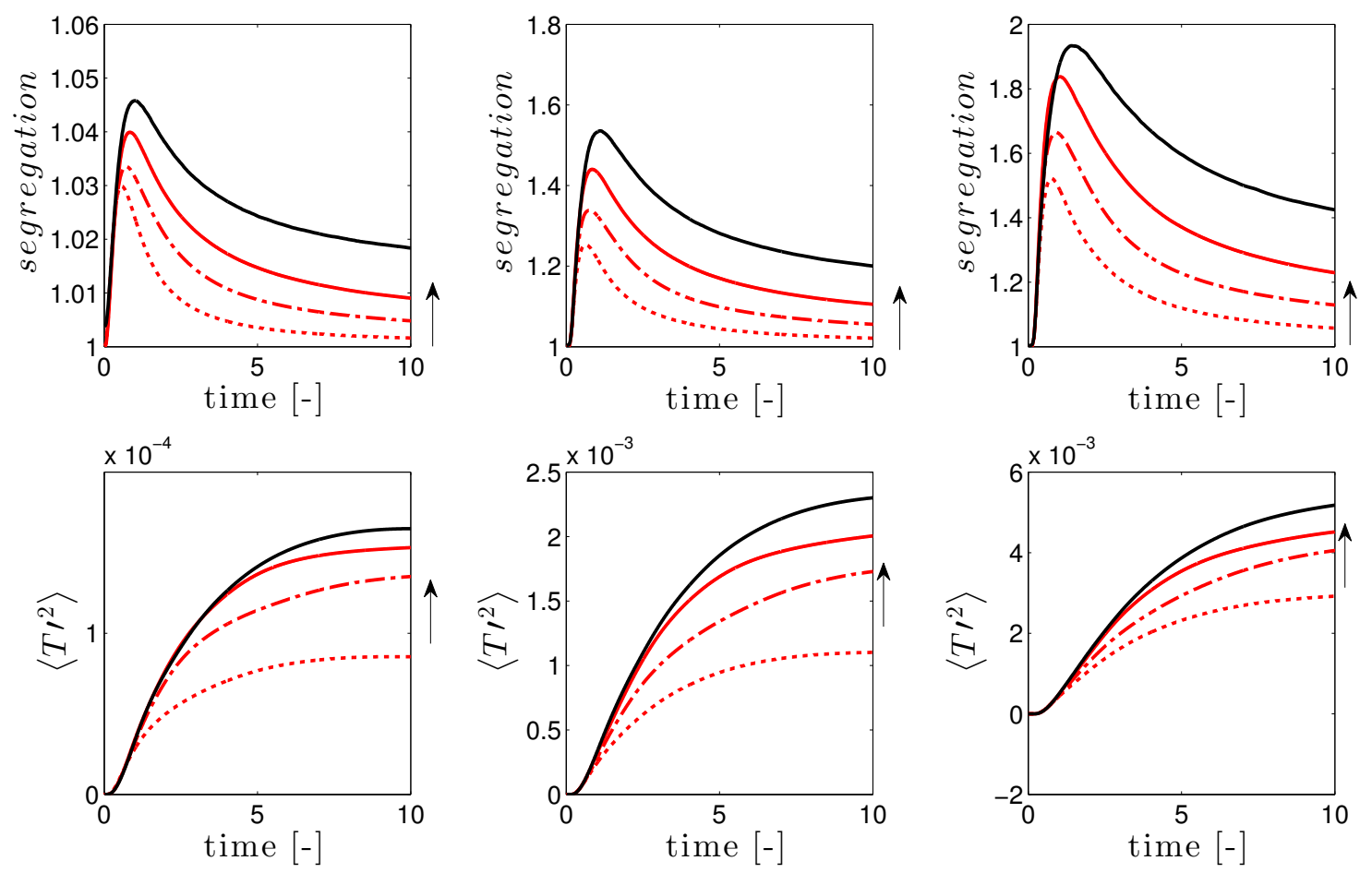

Figure 8: Decaying turbulence with radiation and no gravity: Lagrangianwith 64 particles per cell (black full) versus Eulerian (red) simulations. Segregation (top) and temperature variance (bottom) versus time for $\tau_{p}=0.01,0.05$ and 0.1 . The arrows indicate increasing mesh size from $64^{3}$ to $256^{3}$.

artificial numerical diffusion in the Eulerian method can smear out the particle field to the levels comparable to physical diffusions in the thermal fields. This conclusion is summarized in Fig. 9 by showing maximum segregation and temperature variance as a function of particle relaxation time. The results suggest that there is a direct connection between convergence of segregation and that for temperature variance.

Finally, it is interesting to point out one bias of the present test case: simulations are performed using a constant time step. As the $T K E$ is falling to 0 , the CFL number of both the gas and disperse phase is also dramatically decreasing. The gas phase is weakly sensitive to it, as long as the numerical diffusion is negligible comparing to the "physical" diffusion. This is not the case for the disperse phase concentration for which no "physical" diffusion is involved. Looking at the TKE profile in time, the turbulent velocity and thus the CFL number are divided by 2.5 after $t=1$, leading to a strong increase in numerical diffusion, limiting the segregation and consequently the temperature variance. This trend is confirmed by the fact that all Eulerian results in terms of temperature first follow the Lagrangian reference, and then smoothly diverge. If we look at the results at $t=1$, the Eulerian simulations are definitely close to the Lagrangian reference. This limitation will not be present for the final case, as the turbulence will be sustained, thus leading to a statistically constant CFL number for the whole simulation.

\subsection{Turbulence with radiation and gravity}

\subsubsection{Statistical convergence}

In the final test case, gravity and radiation are active. As explained by Zamansky et al. [52] a buoyancy driven turbulence is triggered, resulting in long-term statistically stationary and homogeneous signals. Mesh convergence study has been carried out for the Lagrangian simulation, ensuring that the level of radiation of the present simulations will not affect the resolution requirements. To analyse these signals, temporal mean of the spatially-averaged statistics are investigated (the signals are given in Appendix A). 

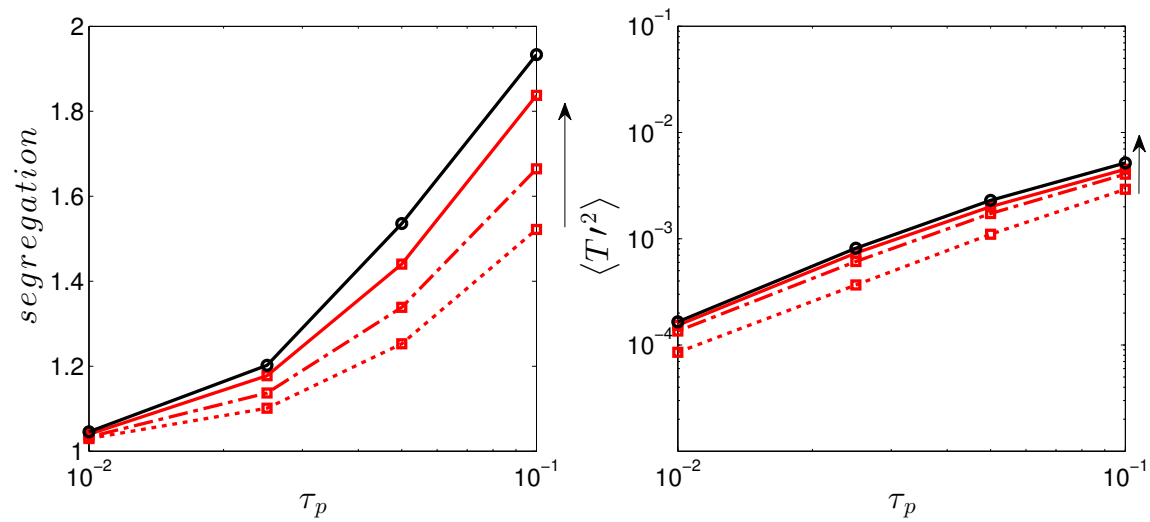

Figure 9: Decaying turbulence with radiation and no gravity: Lagrangian with 64 particles per cell (black full) versus Eulerian (red) simulations. Maximum segregation (left) and temperature variance (right) versus $\tau_{p}$. The arrows indicate increasing mesh size from $64^{3}$ to $256^{3}$.
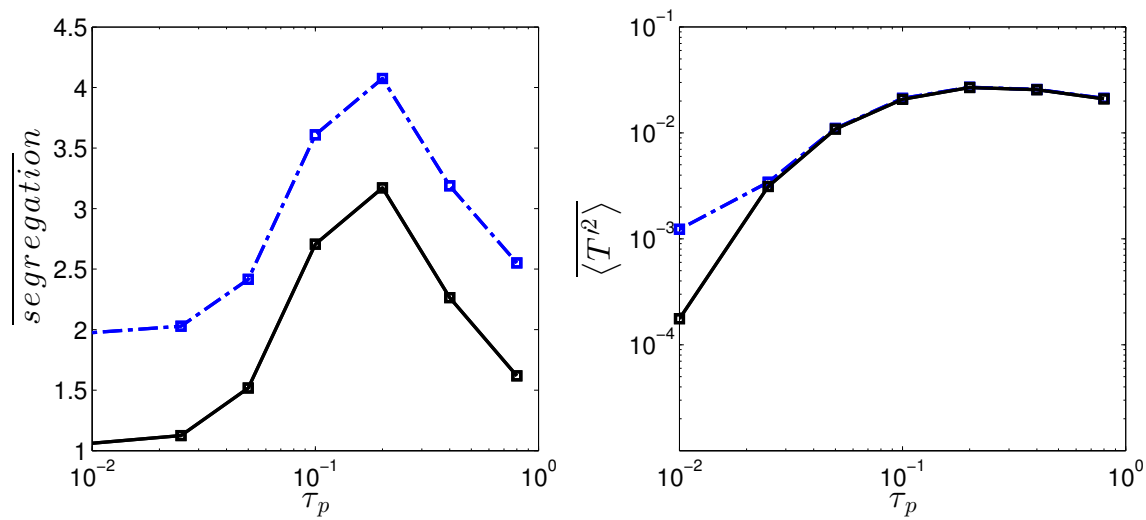

Figure 10: Turbulence with radiation and gravity: effect of the number of particles on the statistics of the Lagrangian simulations. Mean segregation (left) and temperature variance (right) versus $\tau_{p}$, for 1 particle per cell (dot-dashed line) and 256 particles per cell (full line).

Figure 10 shows the averaged segregation and variance of temperature. Similar to previous cases, and as expected, the number of particles has a great effect on the segregation. The number of particles also has some influence on the temperature statistics. The sensitivities and trends with Stokes number are similar to those seen in the case without gravity. This is expected since the nature of temperature transport is similar in both problems.

However, a new sensitivity is observed here which was absent in the previous case. Fig. 11 shows corresponding statistics of TKE and PTKE, and demonstrates that both of these quantities are now to some degree sensitive to the number of particles. This can be explained by the fact that in this system the momentum field is forced by the buoyancy term, which is highly correlated to temperature field. Therefore, convergence of statistics of the momentum field are tied to that of the temperature field. However, due to reasons explained in section 3.2.1 convergence rate is better for systems with higher Stokes number.

Figure 12 shows the mean Kolmogorov time and length scales for different particle relaxation times. This information can be used to determine the range of particle relaxation times for which the long term Stokes number is less than unity as shown by the intersection with the dashed line. Next we examine Eulerian simulations in this range. 

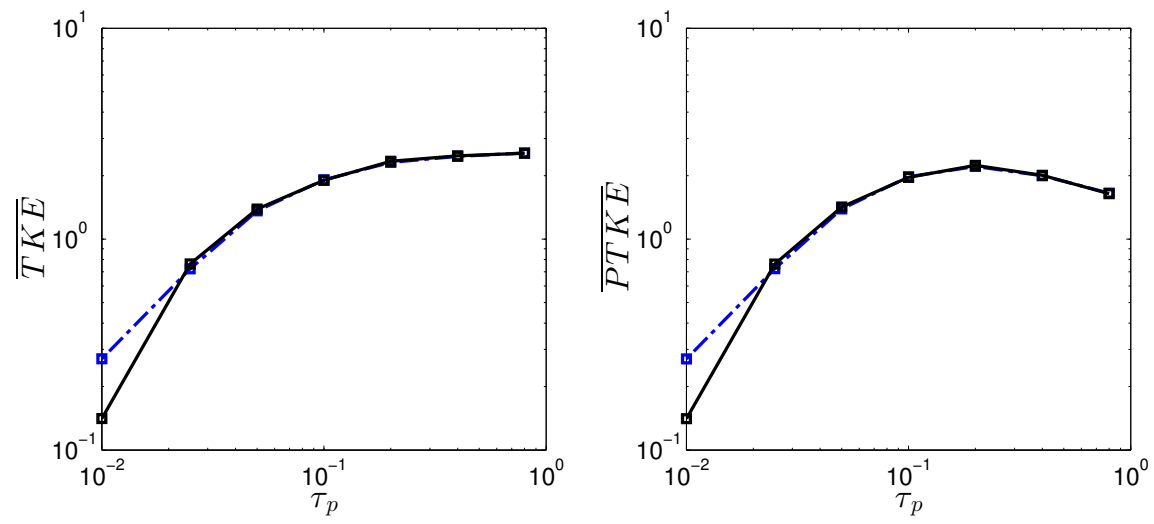

Figure 11: Turbulence with radiation and gravity: effect of the number of particles on the statistics of the Lagrangian simulations. Mean Total Kinetic Energy (left) and Particle Total Kinetic Energy (right) versus $\tau_{p}$, for 1 particle per cell (dot-dashed line) and 256 particles per cell (full line).
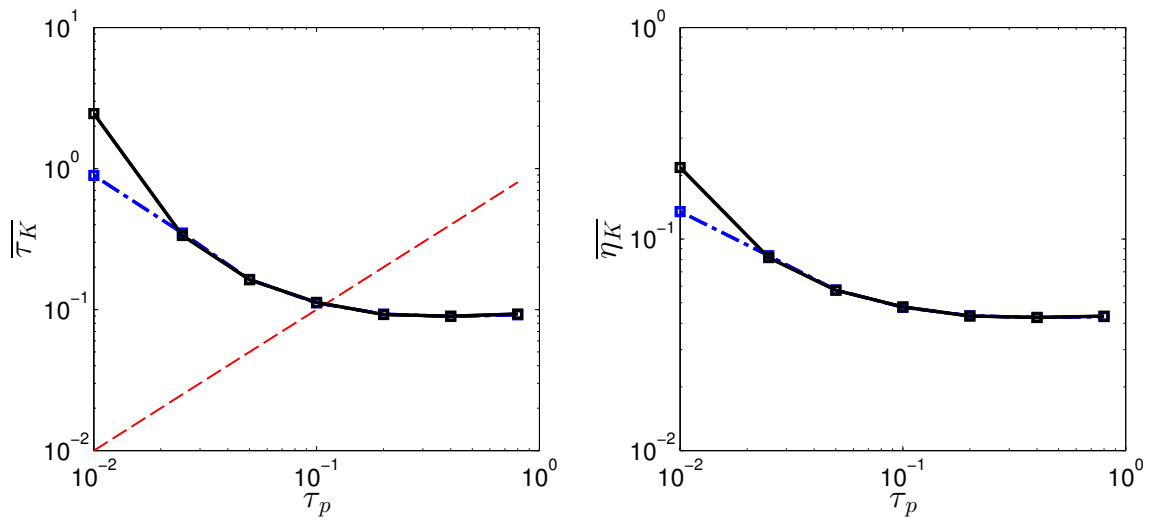

Figure 12: Turbulence with radiation and gravity: effect of the number of particles on the statistics of the Lagrangian simulations. Mean Kolmogorov time (left) and length (right) scales versus $\tau_{p}$, for 1 particle per cell (dot-dashed line) and 256 particles per cell (full line). The red dashed line corresponds to a Stokes number based on the Kolmogorov time scale equal to one. 

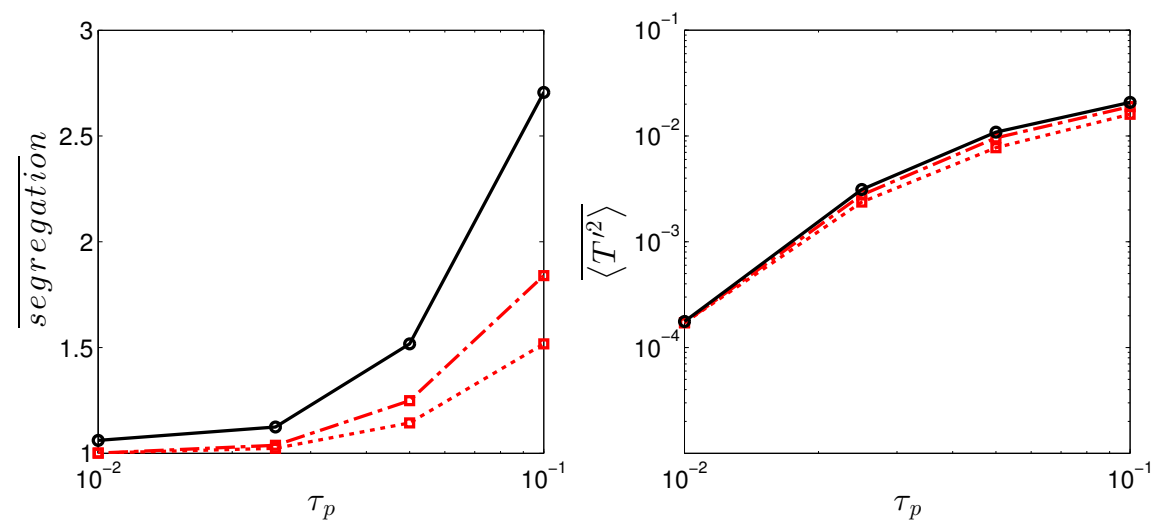

Figure 13: Turbulence with radiation and gravity: Lagrangian with 64 particles per cell (black full) versus Eulerian (red) simulations with $64^{3}$ (dotted line) and $128^{3}$ (dot-dashed line) cells. Mean segregation (left) and temperature variance (right) versus $\tau_{p}$.

\subsubsection{Comparison between Lagrangian and Eulerian results}

Finally, the ability of the Eulerian method to reproduce the Lagrangian results is assessed in this test case in which turbulence itself is coupled with particle segregation. The two previous cases show that the segregation is underestimated by the Eulerian simulation under nominal DNS resolutions. Similarly, the temperature variance is underestimated if the mesh is coarse. In this test case, the temperature field generated by the heat transfer from the disperse phase to the gas triggers turbulence through buoyancy effects. The question is whether the unresolved temperature field will influence the statistics of the generated turbulence.

Our simulations confirmed that the employed Eulerian method can reach a self-sustained turbulence (the signals are given in Appendix A), which is the minimal requirement for assessing the accuracy of the present Eulerian strategy.

Temporally averaged statistics and quantification of their convergence to the reference Lagrangian calculation is assessed in Figs 13-14. Consistent with all previous cases, Fig. 13 indicates that convergence of segregation requires finer resolution than nominal Kolmogorov scale. Regarding temperature statistics, trends are similar to previous case in which buoyancy was inactive: when temperature variance is forced by the particle field, its statistics are sensitive to the mesh resolution due excessive numerical diffusion of the particle field imposed by Eulerian numerics. However, the magnitude of this sensitivity is to some degree less than that in previous case in which the flow field was independent of particles. For example, with the nominal mesh resolution, the Eulerian model leads to at most $20 \%$ error in prediction of temperature variance in the range of particle relaxation times considered here.

Fig. 14 shows the convergence of TKE and PTKE statistics. Again, there is some sensitivity to mesh resolution but the levels are up to about $20 \%$ and less than the sensitivity in segregation. This reduced sensitivity coud have two origins. As mentioned for the case with radiation and no gravity, a possible cause is the fact that the self-sustained state ensures a higher CFL number, thus decreasing the numerical diffusion. It could also suggest that the coupling between turbulence and particle field is more energetic at large scales, which are naturally less sensitive to resolution. While this hypothesis is consistent with previous reports on this phenomenon [52], its confirmation requires more investigation of the physics and nature of clustering in these systems, which is beyond the scope of this report.

Finally the Kolmogorov scales of the generated flows are evaluated in Fig. 15. Results show a good agreement between Lagrangian and Eulerian results. Moreover, we confirm that simulation with relaxation time below 0.1 correspond to Stokes number below one, thus justifying the validity of the Monokinetic closure. 

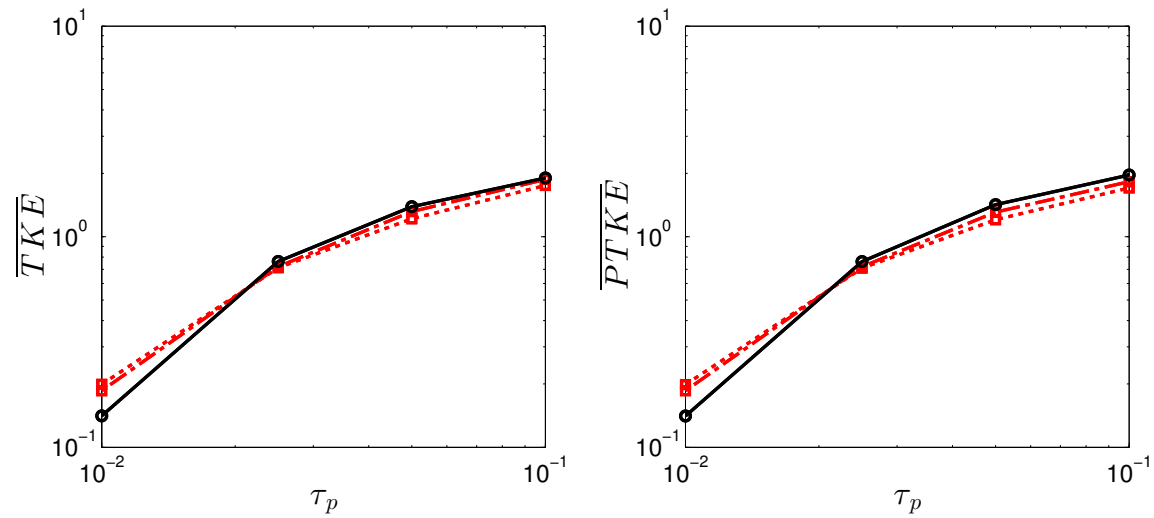

Figure 14: Turbulence with radiation and gravity: Lagrangian with 64 particles per cell (black full) versus Eulerian (red) simulations with $64^{3}$ (dotted line) and $128^{3}$ (dot-dashed line) cells. Mean Total Kinetic Energy (left) and Particle Total Kinetic Energy (right) versus $\tau_{p}$.
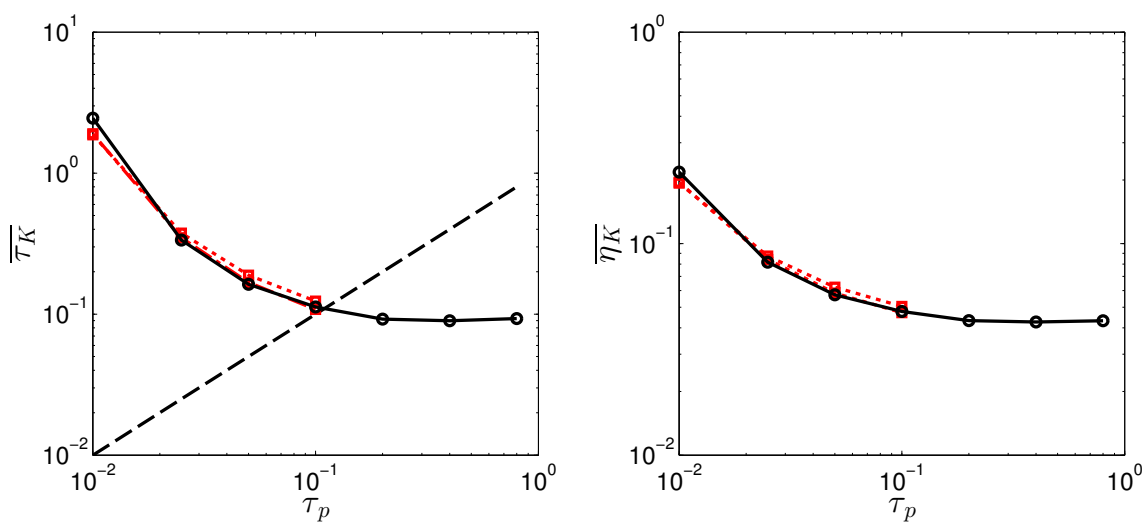

Figure 15: Turbulence with radiation and gravity: Lagrangian with 64 particles per cell (black full) versus Eulerian (red) simulations with $64^{3}$ (dotted line) and $128^{3}$ (dot-dashed line) cells. Kolmogorov time (left) and length (right) scales versus $\tau_{p}$. The dashed line corresponds to a Stokes number based on the Kolmogorov time scale equal to one. 


\section{Summary}

In the present work a comprehensive comparison of Eulerian and Lagrangian strategies for the description of a thermally two-way coupled particle-laden flow has been performed. We considered three model problems with increasing levels of coupling between continuum and disperse phases. In the first problem, we considered homogeneous isotropic decaying turbulence which is unaffected by particles. In the second problem we investigated a temperature field, which is thermally forced by the presence of hot particles, but with minimal impact on the momentum field. In the third problem we studied a scenario in which both temperature and momentum fields are coupled with particles by considering buoyancy effects. For these problems we defined a nominal resolution (or nominal DNS mesh) based on Kolmogorov length scale of the single phase turbulence (uncoupled).

In all scenarios, convergence of segregation statistics was shown to require about 16 particles per nominal DNS mesh, which is mainly a consequence of the convergence rate with respect to the number of particle for the binomial distribution. This is the minimum number of particles needed to justify representing the particle field in the present Eulerian framework ${ }^{3}$. However, our analysis revealed that in order to predict the same segregation statistics via the Monokinetic Eulerian method, resolutions much higher than nominal DNS is needed, about 8 times finer than the nominal carrier phase DNS mesh by extrapolating our results. As a result the number of degrees of freedom required for the Lagrangian method is $60 \%$ the one of the Eulerian simulations. This suggests when it comes to prediction of segregation, use of coarse-grained Lagrangian method is likely to impose lesser computational expense than use of Eulerian methods solved using second order transport scheme. It is then clear that the main limitation of the Eulerian method is the accuracy of the numerics, either for the intrinsic order of the method or for the limiting strategies, as Lagrangian and Eulerian methods are equivalent in the regime we have investigated.

Our analysis was then focused on addressing the question: to what degree errors in an unconverged Eulerian method would influence prediction of continuum fields. We showed that for fields that are not directly coupled with the particles, there is no contamination of errors. However, when a continuum field is forced by the particle field, some sensitivity is observed. The most severe sensitivity was found for the case of radiatively heated particle laden flow (without buoyancy) in which Eulerian method with the nominal DNS resolution was found to underpredict temperature variance by up to about $50 \%$. In the case of buoyancy driven turbulence by hot particles lesser sensitivities were observed in the temperature statistics, but new sensitivities introduced in the momentum prediction, particularly at small Stokes numbers.

As the main limitation of the present Eulerian method is the numerical methods, investigation of higher order convection schemes is a major research axis. Recent results using Discontinuous Galerkin methods have shown a significant cost reduction for accurate segregation prediction [38, 39]. Additional direction would be to employ Eulerian methods with proper moment closure that would allow extension of these analyses to non-monokinetic particle fields that are common at Stokes numbers above unity [23, 47].

\section{Acknowledgments}

This Material is based upon work supported by the Department of Energy National Nuclear Security Administration under Award Number DE-NA0002373-1. The authors also gratefully acknowledge financial support from SAFRAN.

\section{References}

[1] S. V. Apte, K. Mahesh, P. Moin, J. C. Oefelein, Large-eddy simulation of swirling particle-laden flows in a coaxial-jet combustor, Int. J. Multiphase Flow 29 (2003) 1311-1331.

[2] S. Balachandar, A scaling analysis for point-particle approaches to turbulent multiphase flows, International Journal of Multiphase Flow 35 (2009) 801-810.

\footnotetext{
${ }^{3}$ This result does not aim at being an absolute reference and is specific to our simulations, and should be influenced at a lower order by the projection strategies
} 
[3] S. Balachandar, J. Eaton, Turbulent dispersed multiphase flow, Annual Review of Fluid Mechanics 42 (2011) $111-133$.

[4] M. Boivin, O. Simonin, K. Squires, Direct numerical simulation of turbulence modulation by particles in isotropic simulation, J. Fluid Mech. 375 (1998) 235-263.

[5] F. Bouchut, S. Jin, X. Li, Numerical approximations of pressureless and isothermal gas dynamics, SIAM J. Num. Anal. 41 (2003) 135-158.

[6] S. Chapman, T. Cowling, The mathematical theory of non-uniform gazes, Cambridge University Press, 1939.

[7] Y. H. Choi, C. L. Merkle, The application of preconditioning in viscous flows, J. Comp. Phys. 105 (1993) $207-230$.

[8] S. de Chaisemartin, Eulerian models and numerical simulation of turbulent dispersion for polydisperse evaporating sprays, Ph.D. thesis, Ecole Centrale Paris, France, available online at http://tel.archives-ouvertes.fr/tel-00443982/en/ (2009).

[9] O. Druzhinin, S. Elghobashi, Direct numerical simulations of bubble-laden turbulent flows using the two-fluid formulation, Phys. Fluids 10 (1998) 685-697.

[10] J. K. Dukowicz, A particle-fluid numerical model for liquid sprays, J. Comput. Phys. 35 (2) (1980) $229-253$.

[11] J. K. Eaton, J. R. Fessler, Preferential concentration of particles by turbulence, Int. J. Multiphase Flow 20 (1994) $169-209$.

[12] S. Elgobashi, G. Truesdell, Direct simulation of particle dispersion in a decaying isotropic turbulence, Journal of Fluid Mechanics 242 (1992) 655-700.

[13] S. Elgobashi, G. Truesdell, On the two-way interaction between homogeneous turbulence and dispersed solid particles, Physics of Fluids 5(7) (1993) 1790-1801.

[14] J. Ferry, S. Balachandar, A fast Eulerian method for disperse two-phase flow, Int. J. Multiphase Flow 27 (2001) $1199-1226$.

[15] J. Ferry, S. Balachandar, Equilibrium expansion for the eulerian velocity of small particles, Powder Technology 125 (2002) $131-139$.

[16] J. R. Fessler, J. D. Kulick, J. K. Eaton, Preferential concentration of heavy particles in a turbulent channel flow, Phys. Fluid 6 (11) (1994) 3742-3749.

[17] P. Février, O. Simonin, K. D. Squires, Partitioning of particle velocities in gas-solid turbulent flow into a continuous field and a spatially uncorrelated random distribution: theoretical formalism and numerical study, J. Fluid Mech. 533 (2005) $1-46$.

[18] M. Garcia, Development and validation of the Euler-Lagrange formulation on a parallel and unstructured solver for largeeddy simulation, Ph.D. thesis, Université Toulouse III, available online at http://ethesis.inp-toulouse.fr/archive/00000715/ (2009).

[19] R. A. Gore, C. T. Crowe, The effect of particle size on modulating turbulent intensity, Int. J. Multiphase Flow 15 (1989) 279-285.

[20] H. Guillard, C. Viozat, On the behaviour of upwind schemes in the low mach number limit, Comp. Fluids 28 (1998) 63-86.

[21] R. H. A. Ijzermans, E. Meneguz, M. W. Reeks, Segregation of particles in incompressible random flows: singularities, intermittency and random uncorrelated motion, J. Fluid Mech. 653 (2010) 99-136.

[22] A. Kaufmann, M. Moreau, O. Simonin, J. Helie, Comparison between Lagrangian and mesoscopic Eulerian modelling approaches for inertial particles suspended in decaying isotropic turbulence, J. Comput. Phys. 227 (2008) 6448-6472.

[23] F. Laurent, M. Massot, Multi-fluid modeling of laminar poly-dispersed spray flames: origin, assumptions and comparison of the sectional and sampling methods, Combust. Theory and Modelling 5 (2001) 537-572.

[24] F. Laurent, A. Vié, C. Chalons, R. O. Fox, M. Massot, A hierarchy of Eulerian models for trajectory crossing in particleladen turbulent flows over a wide range of Stokes numbers, Annual Research Briefs of the CTR 2012, Center for Turbulence Research, Stanford University (2012) 193-204.

[25] Y. Ling, A. Haselbacher, S. Balachandar, A numerical source of small-scale number-density fluctuations in EulerianLagrangian simulations of multiphase flows, J. Comp. Phys. 229 (5) (2010) 1828-1851.

[26] F. Marble, Dynamics of dusty gases, Annual Review of Fluid Mechanics 2 (1970) 397-446.

[27] E. Masi, Simonin, Algebraic-closure-based moment method for unsteady Eulerian simulations of non-isothermal particleladen turbulent flows at moderate stokes numbers in dilute regime, Flow Turbulence and Combustion 92 (1-2) (2014) $121-145$

[28] E. Masi, O. Simonin, E. Riber, P. Sierra, L. Gicquel, Development of an algebraic-closure-based moment method for unsteady Eulerian simulations of particle-laden turbulent flows in very dilute regime, International Journal of Multiphase Flow 58 (2014) 257-278.

[29] M. Maxey, J. Riley, Equation of motion for a small rigid sphere in a non uniform flow, Physics of Fluids 26 (4) (1983) 2883-2889.

[30] R. Mc Dermott, S. Pope, The parabolic edge reconstruction method (PERM) for Lagrangian particle advection, J. Comp. Phys. 227 (2008) 5447-5491.

[31] J. Minier, On the Lagrangian stochastic methods for turbulent polydisperse two-phase reactive flows, Progress in Energy and Combustion Science 50 (2015) 1-62.

[32] M. Mizukami, R. N. Parthasarathy, G. M. Faeth, Particle-generated turbulence in homogeneous dilute dispersed flows, Int. J. Multiphase Flow 18 (3) (1992) 397-412.

[33] P. Moin, K. Mahesh, Direct Numerical Simulation: A Tool in Turbulence Research, Ann. Rev. Fluid Mech. 30 (1998) 539-578.

[34] T. Passot, A. Pouquet, Numerical simulation of compressible homogeneous flows in turbulent regime, Journal of Fluid Mechanics 181 (1987) 441-466.

[35] N. Patanker, D. Joseph, Modeling and numerical simulation of particulate flows by the Eulerian-Lagrangian approach, Int. J. Multiphase Flow 27 (2001) 1659-1684.

[36] H. Pouransari, A. Mani, On the effects of preferential concentration on heat transfer in particle-based solar receivers, Journal of Fluid Mechanics (in review). 
[37] J. J. Riley, G. S. Patterson, Diffusion experiments with numerically intergrated isotropic turbulence, Physics of Fluids 17 (1974) 292-297.

[38] M. Sabat, A. Larat, A. Vié, M. Massot, Comparison of realizable schemes for the Eulerian simulation of disperse phase flows, Proceedings of FVCA7, Berlin, Germany (2014) 1-8.

[39] M. Sabat, A. Larat, A. Vié, M. Massot, On the development of high order realizable schemes for the Eulerian simulation of disperse phase flows: a convex-state preserving Discontinuous Galerkin method, Journal of Computational Multiphase Flows 6 (3) (2014) 247-270.

[40] U. Saffmann, On the stability of laminar flow of a dusty gas, Journal of Fluid Mechanics 13(1) (1962) 120-128.

[41] M. Sanjosé, J.-M. Senoner, F. Jaegle, B. Cuenot, S. Moreau, T. Poinsot, Fuel injection model for Euler-Euler and EulerLagrange large-eddy simulations of an evaporating spray inside an aeronautical combustor, International Journal of Multiphase Flow 37 (5) (2011) $514-529$.

[42] O. Simonin, Combustion and turbulence in two phase flows. lecture series 1996-02 (1996).

[43] K. Squires, J. Eaton, Measurements of particle dispersion obtained from direct numerical simulations of isotropic turbulence, J. Fluids Mech. 226 (1991) 1-35.

[44] K. D. Squires, J. Eaton, Preferential concentration of particles by turbulence, Physics of Fluids 3 (1991) $1169-1178$.

[45] G. Stokes, On the effect of the inertial friction of fluids on the motions of pendulums, Trans. Cambridge Phil. Soc. 9.

[46] S. Sundaram, L. R. Collins, Collision statistics in an isotropic particle-laden turbulent suspension. part 1. direct numerical simulations, J. Fluid Mech. 335 (1997) 75-109.

[47] A. Vié, F. Doisneau, M. Massot, On the Anisotropic Gaussian closure for the prediction of inertial-particle laden flows, Communications in Computational Physics 17 (1) (2015) 1-46.

[48] L. P. Wang, A. S. Wexler, Y. Zhou, On the collision rate of small particles in isotropic turbulence, ii finite inertia case, Phys. Fluid 10 (10) (1998) 1206-1216.

[49] M. R. Wells, D. E. Stock, The effects of crossing trajectories on the dispersion of particles in a turbulent flow, J. Fluid Mech. 136 (1983) 31-62.

[50] F. A. Williams, Spray combustion and atomization, Phys. Fluids 1 (1958) 541-545.

[51] H. Yoshimoto, S. Goto, Self-similar clustring of inertial particles in homogeneous turbulence, Journal of Fluid Mechanics 577 (2007) 275-286.

[52] R. Zamansky, F. Coletti, M. Massot, A. Mani, Radiation induces turbulence in particle-laden fluids, Physics of Fluids 26 (7) (2014) 1-7.

[53] R. Zamansky, F. Coletti, M. Massot, A. Mani, Turbulent thermal convection driven by heated inertial particles, in consideration for publication in J. Fluid Mech.

\section{Appendix A. Appendix: time signals for case with radiation and gravity}

In this appendix, the time signals for the final case with radiation and gravity are given in Figs. A.18A.19, and shows that this configuration reaches a statistically-stationnary for either Lagrangian or Eulerian simulations, for every Stokes number. 

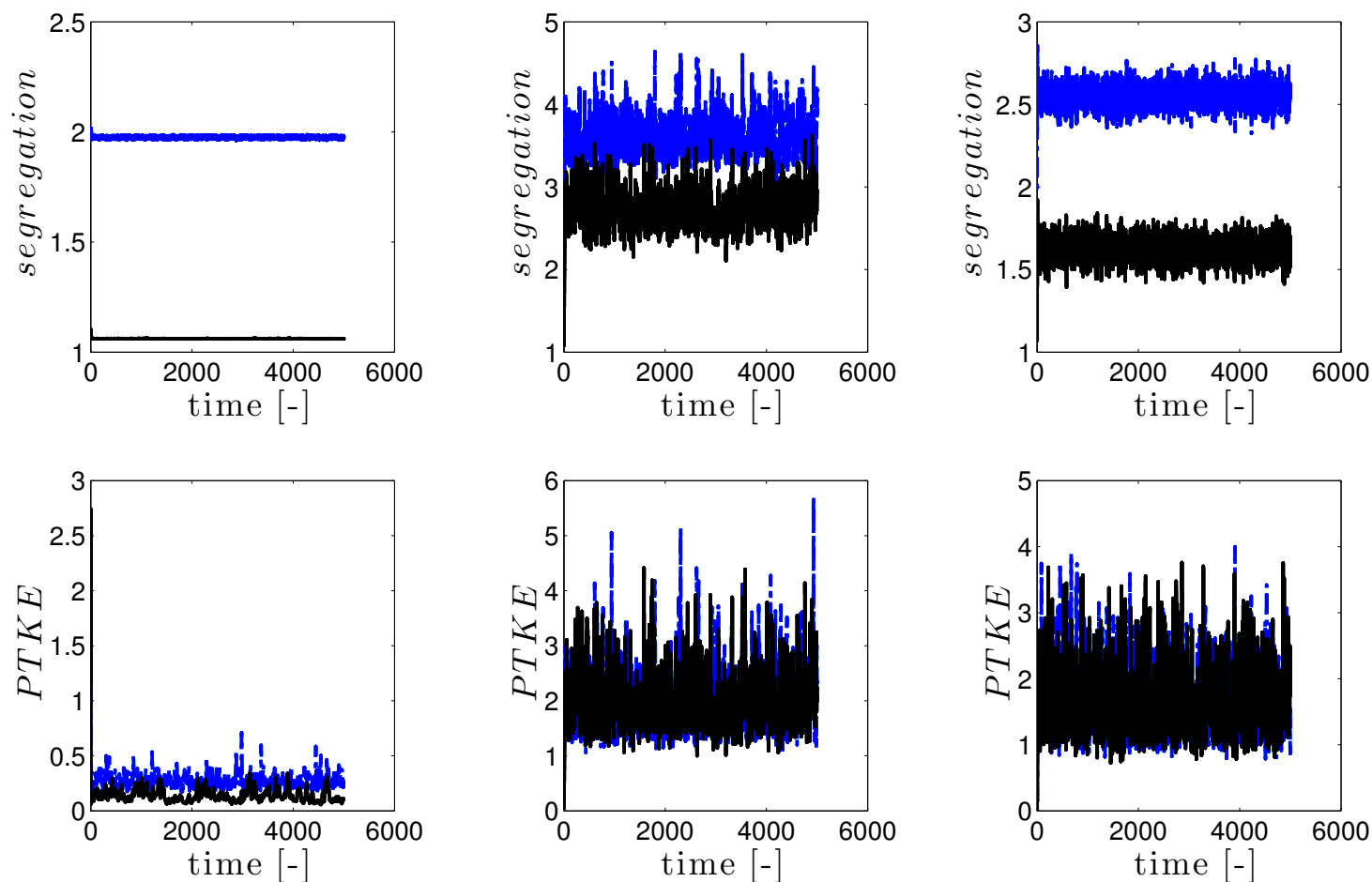

Figure A.16: Turbulence with radiation and gravity: effect of the number of particles on the statistics of the Lagrangian simulations. Segregation (top) and Particle Total Kinetic Energy (bottom) versus time for $\tau_{p}=0.01,0.1$ and 0.8 , for 1 particle per cell (dot-dashed line) and 256 particles per cell (full line).
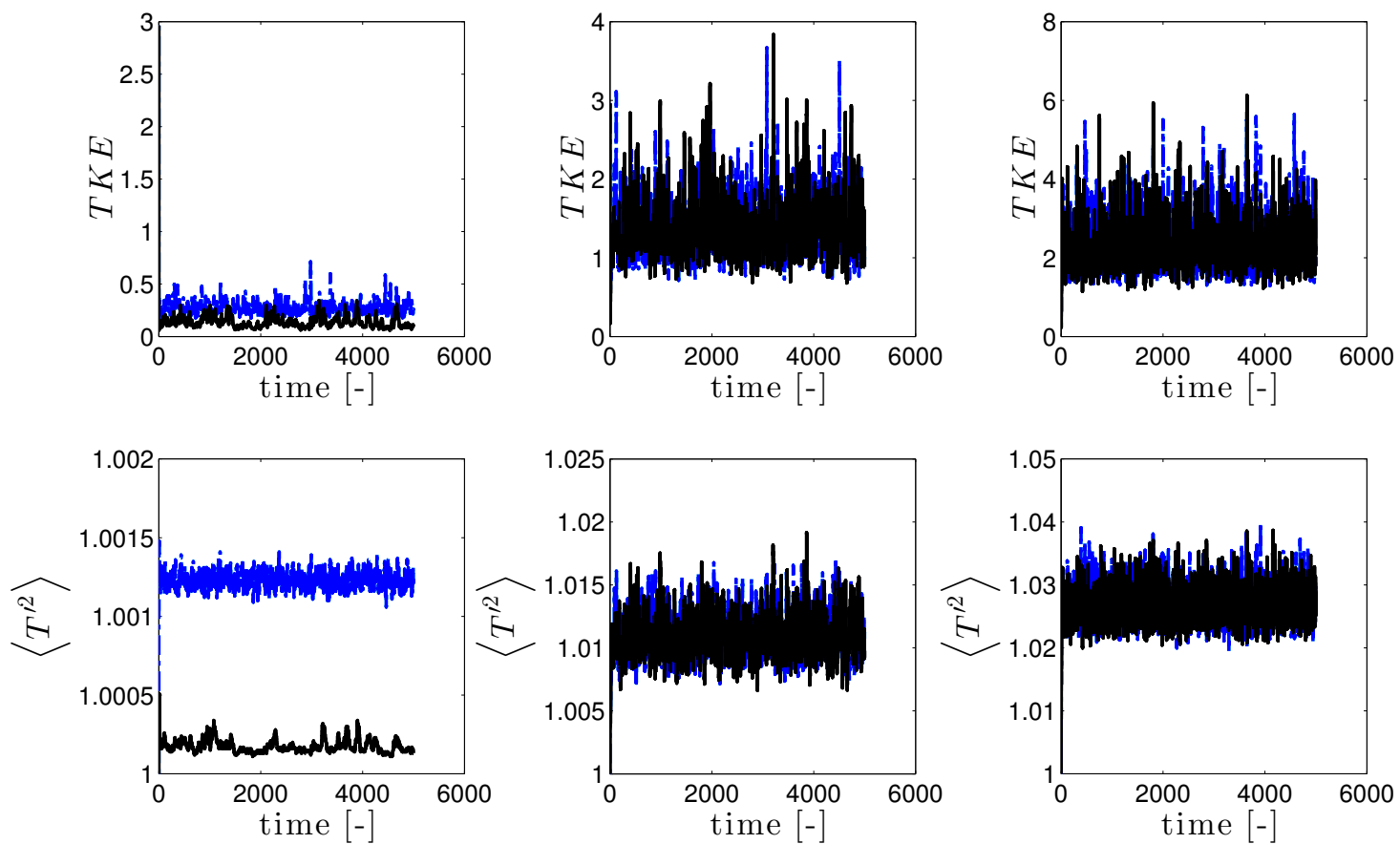

Figure A.17: Turbulence with radiation and gravity: effect of the number of particles on the statistics of the Lagrangian simulations. Total Kinetic Energy (top) and Temperature Variance (bottom) versus time for $\tau_{p}=0.01,0.1$ and 0.8 , for 1 particle per cell (dot-dashed line) and 256 particles per cell (full line). 

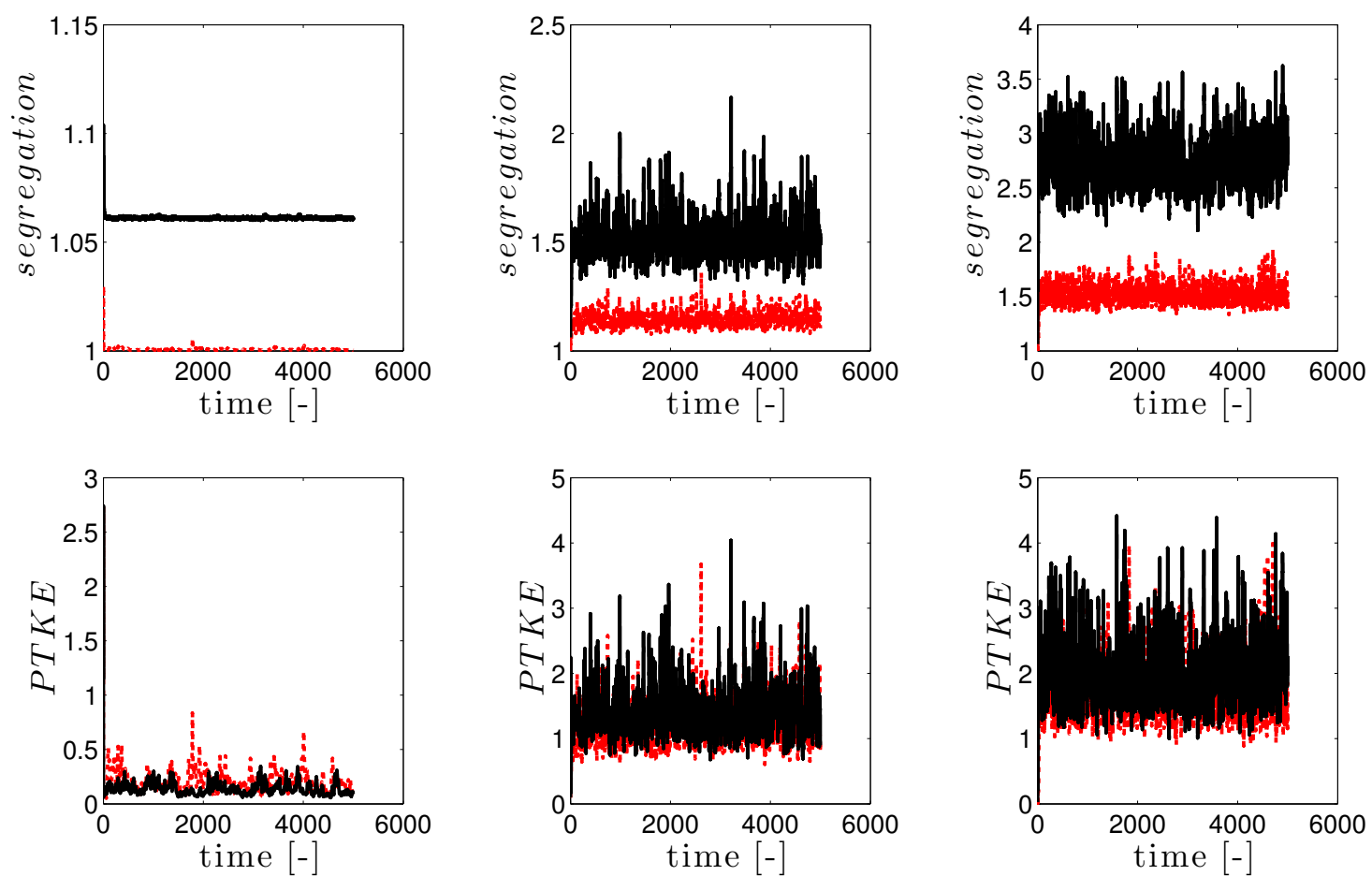

Figure A.18: Turbulence with radiation and gravity: Lagrangian with 64 particles per cell (black full) versus Eulerian (red) simulation with 64 cells. Segregation (top) and Particle Total Kinetic Energy (bottom) versus time for $\tau_{p}=0.01,0.05$ and 0.1 .
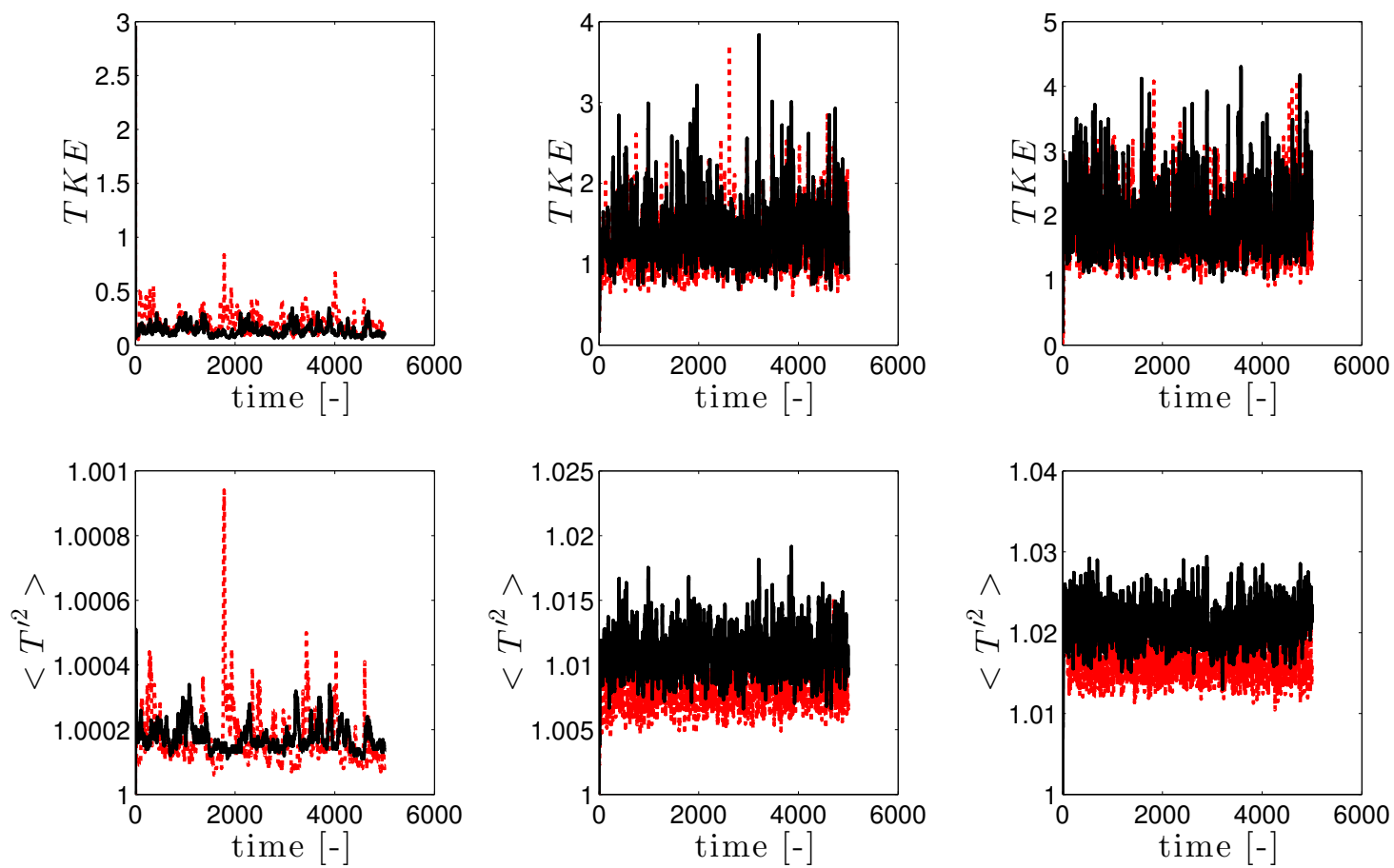

Figure A.19: Turbulence with radiation and gravity: Lagrangianwith 64 particles per cell (black full) versus Eulerian (red) simulation with 64 cells. Total Kinetic Energy (top) and temperature variance (bottom) versus time for $\tau_{p}=0.01,0.05$ and 0.1 . 